\title{
Genome-Wide Characterization and Analysis of CIPK Gene Family in Two Cultivated Allopolyploid Cotton Species: Sequence Variation, Association with Seed Oil Content, and the Role of GhCIPK6
}

\author{
Yupeng Cui ${ }^{1}$, Ying $\mathrm{Su}^{2}$, Junjuan Wang ${ }^{1}$, Bing Jia ${ }^{1}$, Man Wu ${ }^{1}$, Wenfeng Pei ${ }^{1}$, Jinfa Zhang ${ }^{3}$ and \\ Jiwen $\mathrm{Yu}^{1, *(1)}$ \\ 1 State Key Laboratory of Cotton Biology, Institute of Cotton Research of Chinese Academy of Agricultural \\ Sciences, Key Laboratory of Cotton Genetic Improvement, Ministry of Agriculture, Anyang 455000, China; \\ yupeng_cui851026@163.com (Y.C.); Wang20201973@163.com (J.W.); jiabing1814@126.com (B.J.); \\ wuman2004@163.com (M.W.); peiwenfeng1988@163.com (W.P.) \\ 2 Laboratory of Cotton Genetics, Genomics and Breeding, College of Agronomy and Biotechnology/Key \\ Laboratory of Crop Heterosis and Utilization of Ministry of Education/Beijing Key Laboratory of Crop \\ Genetic Improvement, China Agricultural University, Beijing 100193, China; suying0425@163.com \\ 3 Department of Plant and Environmental Sciences, New Mexico State University, Las Cruces, NM 88003, \\ USA; jinzhang@nmsu.edu \\ * Correspondence: yujw666@hotmail.com; Tel.: +86-0372-2562-275
}

Received: 19 December 2019; Accepted: 27 January 2020; Published: 29 January 2020

Abstract: Calcineurin B-like protein-interacting protein kinases (CIPKs), as key regulators, play an important role in plant growth and development and the response to various stresses. In the present study, we identified 80 and 78 CIPK genes in the Gossypium hirsutum and G. barbadense, respectively. The phylogenetic and gene structure analysis divided the cotton CIPK genes into five groups which were classified into an exon-rich clade and an exon-poor clade. A synteny analysis showed that segmental duplication contributed to the expansion of Gossypium CIPK gene family, and purifying selection played a major role in the evolution of the gene family in cotton. Analyses of expression profiles showed that GhCIPK genes had temporal and spatial specificity and could be induced by various abiotic stresses. Fourteen GhCIPK genes were found to contain 17 non-synonymous single nucleotide polymorphisms (SNPs) and co-localized with oil or protein content quantitative trait loci (QTLs). Additionally, five SNPs from four GhCIPKs were found to be significantly associated with oil content in one of the three field tests. Although most GhCIPK genes were not associated with natural variations in cotton oil content, the overexpression of the GhCIPK6 gene reduced the oil content and increased C18:1 and C18:1+C18:1d6 in transgenic cotton as compared to wild-type plants. In addition, we predicted the potential molecular regulatory mechanisms of the GhCIPK genes. In brief, these results enhance our understanding of the roles of CIPK genes in oil synthesis and stress responses.

Keywords: Gossypium; CIPK; various stresses; QTLs; expression and regulation; oil content

\section{Introduction}

Calcium, as a secondary messenger, plays an important role in plant growth and development and plant responses to environmental stresses [1]. There are four major calcium ion sensors in plants, including calmodulin, calmodulin-like protein, calcium-dependent protein kinase, and calcineurin B-like protein (CBL), which sense and decode the changes of calcium ion concentration in response to various stimuli [2]. Among the four calcium sensors, CBL decodes calcium transients by interacting with and modulating the activity of CBL-interacting protein kinases (CIPKs) in higher plants [3]. 
CIPKs belong to the sucrose nonfermenting 1 (SNF1) kinase family in plants, which contain three families: SnRK1, SnRK2, and SnRK3, and CIPKs are also known as the SnRK3 family [4,5]. Commonly, CIPK proteins contain a conserved kinase domain in the $\mathrm{N}$-terminus and a regulatory domain in the C-terminus, which is separated by a variable junction domain. The N-terminal domain of CIPKs is similar with the protein structures of SNF1 kinase and AMO-dependent protein kinase. A conserved NAF domain within the C-terminus of CIPKs is required for interacting with CBLs, which activate the catalytic activity of CIPKs, and the activated CIPKs can transfer perceived calcium signals by phosphorylating target downstream signaling components [6,7]. Moreover, a protein phosphatase interaction (PPI) domain in the C-terminus of CIPKs can also interact with specific members of protein phosphatase 2C (PP2C) [8].

Following the identification of 26 CIPK family genes in Arabidopsis thaliana genome [9], CIPK genes in many plant species have also been identified by genome-wide analyses of their genomes. For example, there are 34 in rice (Oryza sativa) [10], 43 in maize (Zea mays) [11], 27 in poplar (Populus tremula) [12], 23 in Brassica napus [13], 52 in soybean (Glycine max) [14], 20 in Vitis vinifera [15], 34 in apple (Malus domestica) [16], and 16 CIPKs in Prunus mume [17]. These studies reported that the CIPK gene family can be classified into two groups, including an exon-rich group and exon-poor group. It was reported that CIPK genes participate in plant growth and development, and play critical roles in various stresses, including abiotic stresses and hormones $[15,18]$. The expression levels of CIPK genes in G. raimondii and G. arboreum were induced under abiotic stresses (drought, salt and low temperature) [19]. In Arabidopsis, the expression level of AtCIPK3 was enhanced during the early developmental stages of seedlings, and the Atcipk3 mutant showed high sensitivity under abscisic acid (ABA) stress during seed germination [20], and overexpression of GhCIPK6 increased tolerance to salt, drought, and ABA stresses in transgenic Arabidopsis [21]. Overexpression of TaCIPK24 from wheat (Triticum aestivum L.) improves salt tolerance and enhances reactive oxygen species (ROS) scavenging capacity in transgenic Arabidopsis [18]. The overexpression of ZmCIPK8 from Zea mays confers drought tolerance in transgenic tobacco [22], and overexpression of TaCIPK27 increases tolerance to drought, but decreases sensitivity to ABA treatment in transgenic Arabidopsis [23]. Overexpression of each of the $12 \mathrm{HsCIPKs}$ from barley (Hordeum spontaneum) in rice shows that all but HsCIPK28 enhance the tolerance of roots to multiple heavy metal toxicities, and eight HsCIPKs (except for HsCIPK9, HsCIPK11, HsCIPK14, and HsCIPK24) improve the tolerance to salt, drought, and ABA [24]. Overexpressing the LlaCIPK gene from Lepidium can enhance cold tolerance in tobacco by increasing the level of proline and cell membrane stability [25]. Although the CIPK gene family was reported in G. arboreum and G. raimondii [19], little is known about their detailed information in G. hirsutum and G. barbadense, especially their potential roles in various abiotic stresses and cottonseed oil regulation.

Cotton (Gossypium) is one of the most important fiber crops around the world, and cottonseed is the main byproduct of cotton, with the oil content of cottonseed ranging from $28.24 \%$ to $44.05 \%$ and unsaturated fatty acids accounting for the largest component of the oil [26,27]. To explore the genetic composition of oil content is helpful for utilizing cottonseed. Although there are many studies in genetic analysis and quantitative trait locus (QTL) mapping of oil content in different cotton populations [26,28], reports on cotton CIPK proteins are minimal. In B. napus, BnCIPK9 was identified as involved in oil content regulation in $\mathrm{F}_{2}$ population; overexpression of BnCIPK9 in B. napus can decrease seed oil content, and seed oil contents of Atcipk9 mutants were significantly higher than that of wild-type (WT) plants [29]. These results suggest that CIPK genes play important roles in carbohydrate and energy metabolism, and oil synthesis. Recently, the whole-genome sequences of four cotton species, including G. raimondii [27,30], G. arboreum [31,32], G. hirsutum acc. TM-1 [33-36], and G. barbadense, acc. 3-79, cv. Xinhai 21 and cv. H7124 [35-38], provide an opportunity to systematically identify CIPK genes in cotton.

In this study, we identified CIPK genes from G. hirsutum and G. barbadense. The identified CIPKs were comprehensively analyzed for phylogenetic classifications, gene structures, protein motifs, chromosomal locations, and duplicated genes. Subsequently, gene expression profiles of GhCIPK genes 
were analyzed in different tissues and various abiotic stresses. Finally, the co-localization of GhCIPK genes with quantitative trait loci (QTLs) for seed oil or protein content, and sequence variations were analyzed, and the primary function of GhCIPK6 was also investigated in cottonseed oil synthesis. In addition, alternative splicing events and miRNA target sites of GhCIPKs were also predicted. Our study provides a solid foundation for further study of the roles of cotton CIPK genes in cotton growth and development, stress responses, and oil synthesis.

\section{Results}

\subsection{Identification of CIPK Genes in G. hirsutum and G. barbadense}

To identify the CIPK genes in G. hirsutum and G. barbadense, we conducted a BLASTP against two cotton genomes with CIPK protein sequences of Arabidopsis (26) and rice (34) as queries (Table S1). Then, the InterProscan and SMART databases were used to further verify the putative CIPK proteins. Finally, the two steps resulted in 80 CIPK genes from G. hirsutum Nanjing Agricultural University (NAU) version, 79 CIPK genes from G. hirsutum Zhejiang University (ZJU) version, and 79 CIPK genes from G. hirsutum Huazhong Agricultural University (HAU) version (Table S2). A total of 72, 78, and 77 CIPK genes were identified in G. barbadense cv. Xinhai 21 genome (NAU version), acc. 3-79 genome (HAU version), and cv. H7124 genome (ZJU version), respectively, using the same methods (Table S3). Information about the protein kinase domain and the NAF domain in each cotton CIPK protein of G. hirsutum and G. barbadense is listed in Table S4. Combining these results, 80 GhCIPKs from G. hirsutum NAU version and 78 GbCIPKs from G. barbadense HAU version were analyzed in the subsequent research.

These cotton CIPK genes were named GhCIPK1-GhCIPK80 and GbCIPK1-GbCIPK78 in G. hirsutum and G. barbadense, respectively, according to their chromosomal positions. The gene name, locus IDs, genomics positions, and other features are shown in Tables S2 and S3. Two genomes of allotetraploid cotton species (G. hirsutum and G. barbadense) underwent a diploidization following the divergence of G. arboreum (A genome) and G. raimondii (D genome). Based on the genome scans of two diploid cotton species, the CIPK gene family was investigated in G. raimondii (41) and G. arboreum (39) [19]. To clarify the divergence during cotton evolution, we analyzed the orthologous CIPK genes between $G$. raimondii, G. arboreum, and the A and D subgenomes of the two allotetraploid species (G. hirsutum and G. barbadense) (Table S5, Figure S1). In G. hirsutum, a total of 37 CIPK genes in the D subgenome had orthologs in the D genome, and 35 genes in the A subgenome had orthologs in the A genome. In G. barbadense, 34 CIPK genes in the A subgenome had orthologs in the A genome, while 40 genes in the D subgenome had orthologs in $\mathrm{D}$ genome. This revealed that the collinear relationship of CIPK genes between Dt subgenome and D genome was higher than the At subgenome and A genome, suggesting that more CIPK genes were lost in the At subgenome of G. hirsutum or G. barbadense during evolution. A total of 71 CIPK genes in G. hirsutum had orthologs in the G. barbadense, and 39 pairs of orthologous genes between G. raimondii and G. arboreum (Table S5, Figure S1). Additionally, a total of 39 and 38 pairs of homoeologs for CIPK genes was identified in G. hirsutum and G. barbadense, respectively (Table S6, Figure S2), since each pair had one gene from the At subgenome and another from the Dt subgenome.

\subsection{Phylogenetic Classifications, Structural Features, and Conserved Motifs Analysis of Cotton CIPK Genes}

The evolutionary relationship of CIPK genes from G. hirsutum (80), G. barbadense (78), Arabidopsis (26), and rice (34) is shown in Figure 1. A total of 218 CIPKs was divided into five groups (I, II, III, IV, and V), consistent with previous reports of CIPKs in Arabidopsis and poplar [12]. Group I consisted of 55 cotton CIPK genes (28 G. hirsutum and 27 G. barbadense CIPK genes), as compared to 9 Arabidopsis and 12 rice CIPK genes. Groups II and V contained an equal number of cotton CIPKs, with 6 G. hirsutum and 6 G. barbadense CIPK genes, as compared to 2 and 4 CIPK genes for Groups II and V, respectively, each from Arabidopsis and rice. Group III is the largest and was composed of 84 CIPK genes, including 32 from G. hirsutum, 31 from G. barbadense, 8 from Arabidopsis, and 13 from 
rice. Group IV contained 8 G. hirsutum and 8 G. barbadense CIPK genes, as compared to 3 CIPK genes each from Arabidopsis and rice. In the phylogenetic tree, G. hirsutum and G. barbadense contained more CIPK genes than Arabidopsis and rice in each group, and cotton CIPK genes had closer relationships with $A t C I P K s$ than with OsCIPKs, which is consistent with our understanding of the evolutionary history of these genes in plants. From the view of evolution, one CIPK gene in G. raimondii corresponds with one orthologous gene in G. arboreum and two orthologous genes in G. hirsutum and G. barbadense (Figures S3 and S4), since the two diploid genomes of G. raimondii and G. arboreum are the progenitors for G. hirsutum and G. barbadense [27]. Most CIPK genes exhibit such a phylogenetic correspondence in G. arboreum, G. raimondii, G. hirsutum, and G. barbadense. As highlighted in Figure S3, two CIPK genes (GrCIPK7 and GrCIPK39) from G. raimondii showed a one-to-one correspondence with two (GhCIPK63 and GhCIPK71) in the D subgenome of G. hirsutum. However, there were no corresponding CIPK genes in G. arboreum for the two genes (GhCIPK24 and GhCIPK32) from A subgenome of G. hirsutum, suggesting that two genes were lost in G. arboreum after the divergence from G. raimondii. Futhermore, we observed GaCIPK12 and GrCIPK18 lost corresponding genes in G. barbadense, and there was no corresponding gene in G. raimondii for GbCIPK7 in the D subgenome of G. barbadense, and a similar phenomenon was also observed in GbCIPK24 (Figure S4). Additionally, three genes (GaCIPK12, GaCIPK19, and GaCIPK37) in G. arboreum only had corresponding genes in G. hirsutum, and GrCIPK32 only had one corresponding gene in G. barbadense (Figures S3 and S4).

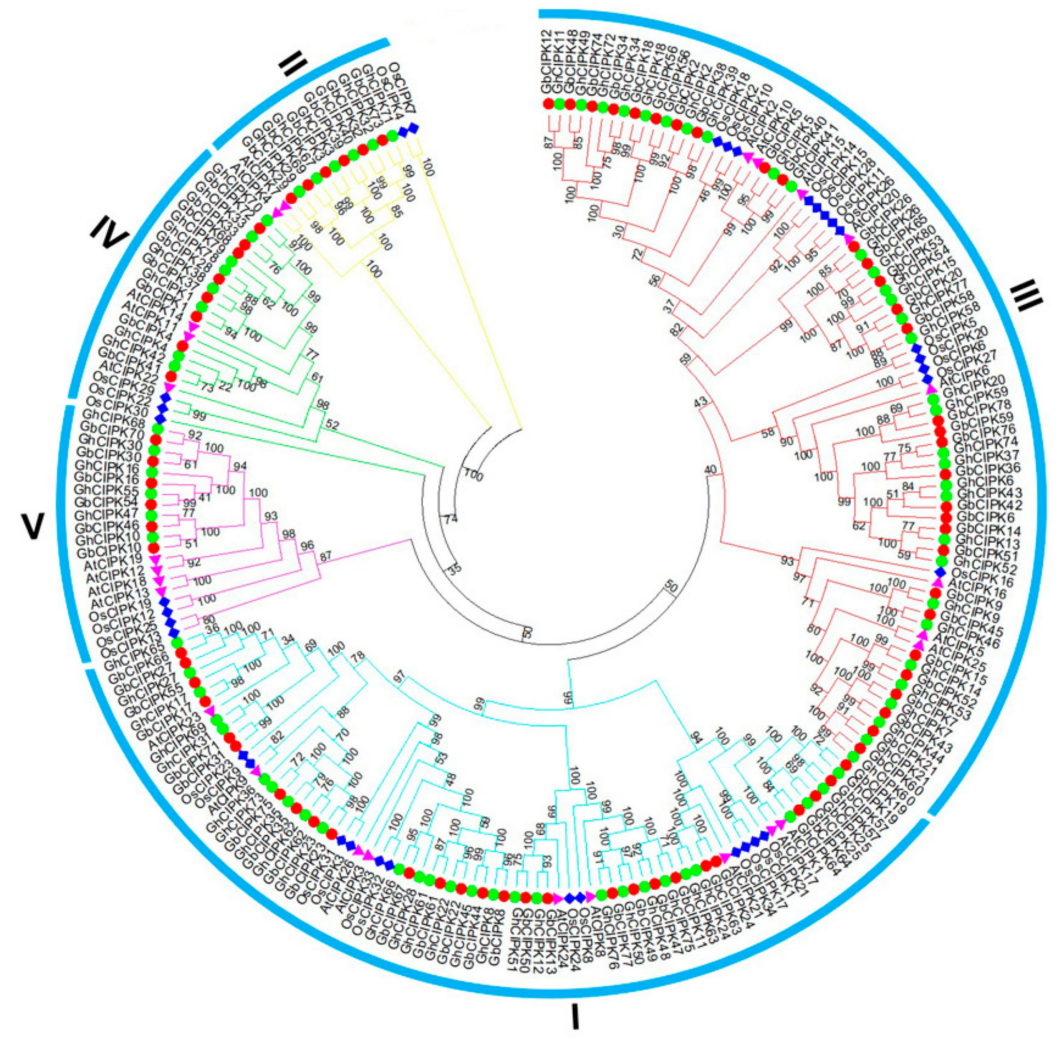

Figure 1. Phylogenetic relationships of calcineurin B-like protein-interacting protein kinases (CIPKs) from G. hirsutum, G. barbadense, Arabidopsis, and rice. The unrooted phylogenetic tree was constructed with MEGA 5.0 using the neighbor-joining method, and bootstrap analysis was performed with 1000 replicates. The CIPKs from G. hirsutum, G. barbadense, Arabidopsis, and rice are marked with green dots, red dots, magenta triangles, and blue rhombus, respectively.

We further analyzed the CIPKs gene structure from G. hirsutum and G. barbadense (Figure 2). Our results show that these CIPK genes are clearly divided into an exon-rich clade ( $>9$ exons per gene) and an exon-poor clade ( $<3$ exons per gene). The exon-rich clade members containing 10 to 16 exons were clustered to Group I, while the exon-poor clade members containing 1 to 3 exons were clustered to the 
other four groups (Group II, III, IV, and V; Figure 2). Gene length in the exon-rich clade was longer than that in the exon-poor clade. The code length of gene members in the exon-rich clade ranged from 4355 to $8597 \mathrm{bp}$, while in the exon-poor clade, it ranged from 1223 to $2799 \mathrm{bp}$, except for GhCIPK34, GhCIPK78, GbCIPK41, GbCIPK20, GhCIPK80, and GhCIPK26, which contained 662, 992, 3117, 3127, 3154, and $3156 \mathrm{bp}$, respectively. Thus, consistent with the phylogenetic tree in Figure 1, the gene structures of GhCIPKs and GbCIPKs were highly similar in the same group. For example, all the cotton CIPK genes (6 GbCIPKs and 6 GhCIPKs) in Group II contained only one exon, and 15 of 16 cotton CIPK genes (7 GbCIPKs and 8 GhCIPKs) in Group IV contained one exon.

Conserved motifs were also scanned in these CIPK proteins using the MEME online tool. A total of 18 motifs were identified (Figure S5), and the details of each motif are shown in Figure S6. All cotton CIPK proteins contained motif 13, which was annotated as the NAF domain, except GhCIPK36. GhCIPK5, GhCIPK41, GbCIPK5, and GbCIPK40 did not present motif 13, but they contained the amino acid sequence of the NAF domain (Figure S7). Additionally, motifs 16 and 17 were only presented in Group II, suggesting that they might perform group specific functions. 


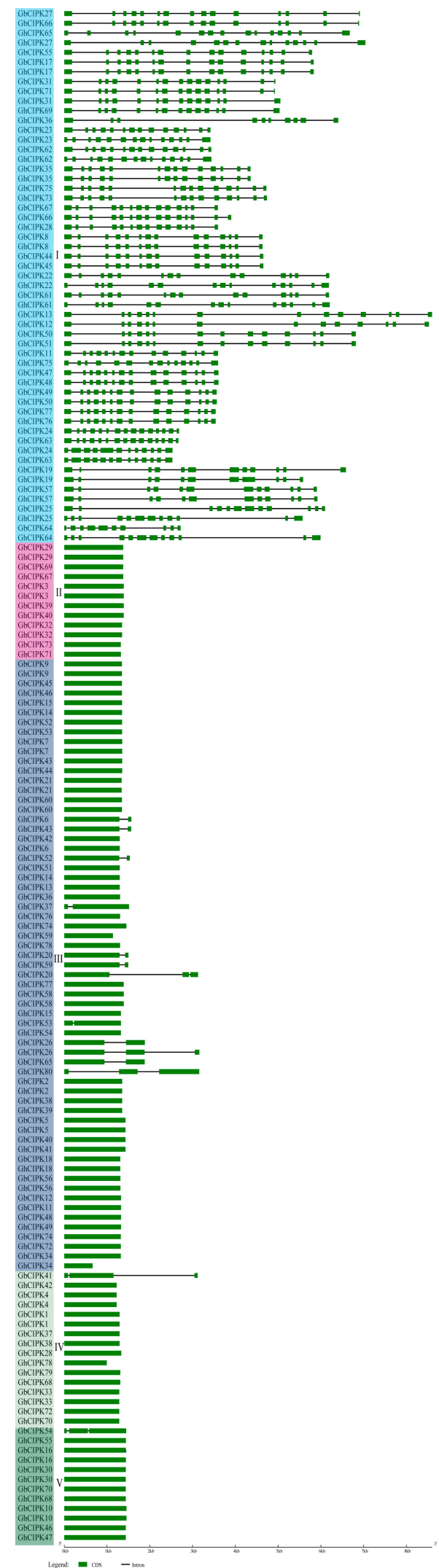

Figure 2. The gene structure of CIPK genes in G. hirsutum and G. barbadense. Introns are presented by black lines and exons by green boxes. 


\subsection{Chromosomal Location and Gene Duplication of CIPK Genes in Two Gossypium Species}

The 80 GhCIPKs were mapped to their corresponding chromosomes (Table S2, Figure 3A), and all of them were unevenly distributed on the chromosomes of G. hirsutum. For example, all 80 GhCIPKs were distributed on 21 of 26 chromosomes, except for A01, A04, D01, D04, and D12. Chromosome D06 contained the largest member (9) of GhCIPKs among all upland cotton chromosomes, while chromosomes A11, A12, A13, D11, and D13 each contained only one GhCIPK gene (Figure 3A). We also mapped the GbCIPKs from G. barbadense and found that all 78 GbCIPKs were also unevenly distributed on their chromosomes (Table S3, Figure 3B). All 78 GbCIPKs were found on 20 of 26 chromosomes, and D06 also contained the largest member (10) of GbCIPKs, followed by A06 with eight GbCIPKs (Figure 3B).
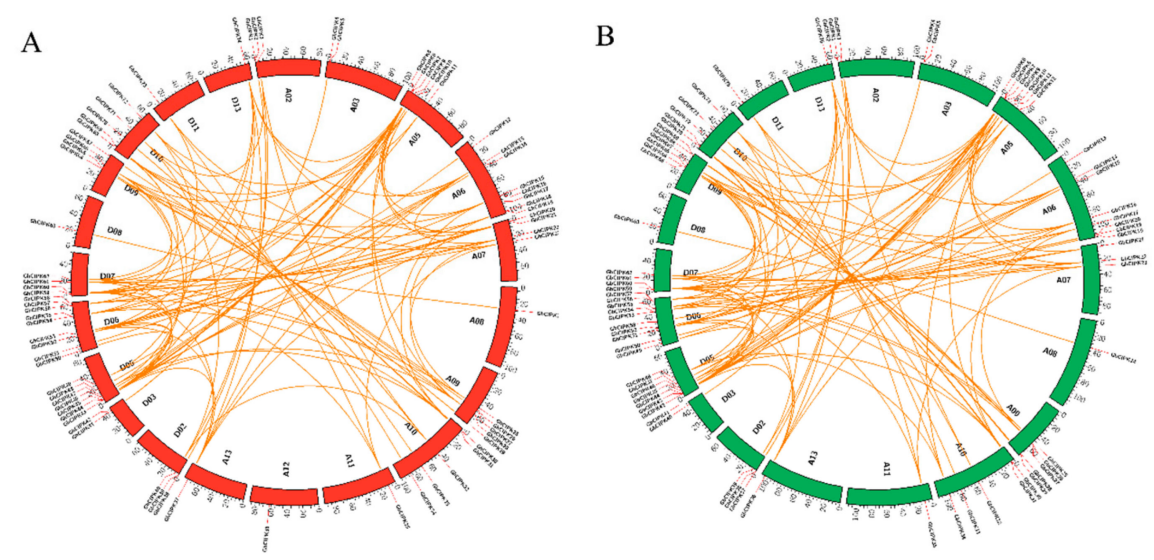

Figure 3. Genomic localization and gene duplication of CIPK genes in G. hirsutum and G. barbadense. (A) Genomic localization and gene duplication of CIPK genes in G. hirsutum; (B) Genomic localization and gene duplication of CIPK genes in G. barbadense. Each of the duplicated gene pairs in GhCIPKs and GbCIPKs is connected with orange color lines. Chromosomes in G. hirsutumand G. barbadense are marked with red and green, respectively.

To elucidate the driving force for the evolution and the functional divergence of CIPK genes, the occurrence of duplication events of cotton CIPK genes was analyzed. As shown in Figure 3 and Table S7, 109 and 107 duplicated gene pairs of CIPK genes were identified in G. hirsutum and G. barbadense, respectively. All were involved in segmental duplication, since these duplicated gene pairs were located on different chromosomes. In addition, there were a series of several duplication events in the two cotton species, for example, GhCIPK11/GhCIPK49, GhCIPK11/GhCIPK72, GbCIPK34/GbCIPK48, and GbCIPK34/GbCIPK74. These results suggest that segmental duplication events contributed to the CIPK gene family in both of G. hirsutum and G. barbadense. We further calculated the $\mathrm{Ka} / \mathrm{Ks}$ ratio to explore the selective constraints on each pair of duplicated CIPK gene in G. hirsutum and G. barbadense (Table 1). In G. hirsutum, 85 of 109 duplicated GhCIPK gene pairs had a Ka/Ks ratio $<1$, which demonstrates that these genes had undergone strong purifying selection pressure; one gene pair (GhCIPK9/GhCIPK46) had a Ka/Ks ratio $>1$, indicating that this gene might have undergone positive selection; the remaining 24 duplicated pairs with ratios $=1$ seemed to be under neutral selection. In G. barbadense, almost all GbCIPK duplicated pairs had a Ka/Ks ratio <1, three pairs (GbCIPK8/GbCIPK44, GbCIPK10/GbCIPK46, and GbCIPK36/GbCIPK76) had a Ka/Ks ratio >1, and three pairs (GbCIPK13/GbCIPK50, GbCIPK14/GbCIPK51, and $G b C I P K 59 / G b C I P K 78$ ) had a Ka/Ks ratio $=1$. These results suggested that the function of the duplicated CIPK genes in G. hirsutum and G. barbadense did not diverge much during subsequent evolution, and purifying selection could mainly contribute to the maintenance of function in the two Gossypium CIPK gene families. 
Table 1. Ka/Ks analysis for the duplicated CIPK gene pairs in G. hirsutum and G. barbadense.

\begin{tabular}{|c|c|c|c|c|c|c|c|}
\hline Species & $\begin{array}{c}\text { Duplicated } \\
\text { Gene } 1\end{array}$ & $\begin{array}{c}\text { Duplicated } \\
\text { Gene } 2\end{array}$ & Ka & Ks & $\mathrm{Ka} / \mathrm{Ks}$ & $\begin{array}{l}\text { Purifying } \\
\text { Selection }\end{array}$ & $\begin{array}{c}\text { Duplicate } \\
\text { Type }\end{array}$ \\
\hline \multirow[t]{58}{*}{ G. hirsutum } & GhCIPK1 & GhCIPK38 & 0 & 0.0296 & 0 & No & Segmental \\
\hline & GhCIPK10 & GhCIPK47 & 0.0081 & 0 & 0 & No & Segmental \\
\hline & GhCIPK10 & GhCIPK30 & 0.0985 & 0.7646 & 0.1288 & Yes & Segmental \\
\hline & GhCIPK10 & GhCIPK68 & 0.0985 & 0.7646 & 0.1288 & Yes & Segmental \\
\hline & GhCIPK10 & GhCIPK55 & 0.0983 & 0.3954 & 0.2486 & Yes & Segmental \\
\hline & GhCIPK10 & GhCIPK16 & 0.0983 & 0.3474 & 0.283 & Yes & Segmental \\
\hline & GhCIPK11 & GhCIPK49 & 0.0165 & 0.0282 & 0.5851 & Yes & Segmental \\
\hline & GhCIPK11 & GhCIPK72 & 0.1436 & 0.5354 & 0.2682 & Yes & Segmental \\
\hline & GhCIPK12 & GhCIPK51 & 0 & 0.0619 & 0 & No & Segmental \\
\hline & GhCIPK13 & GhCIPK20 & 0.0905 & 0.7899 & 0.1146 & Yes & Segmental \\
\hline & GhCIPK13 & GhCIPK37 & 0.0959 & 0.7194 & 0.1333 & Yes & Segmental \\
\hline & GhCIPK13 & GhCIPK43 & 0.0822 & 0.9098 & 0.0903 & Yes & Segmental \\
\hline & GhCIPK13 & GhCIPK52 & 0 & 0 & 0 & No & Segmental \\
\hline & GhCIPK13 & GhCIPK59 & 0.0997 & 0.7138 & 0.1397 & Yes & Segmental \\
\hline & GhCIPK13 & GhCIPK74 & 0.1052 & 0.5893 & 0.1785 & Yes & Segmental \\
\hline & GhCIPK14 & GhCIPK53 & 0.0242 & 0.031 & 0.7806 & Yes & Segmental \\
\hline & GhCIPK15 & GhCIPK54 & 0.0163 & 0.0294 & 0.5544 & Yes & Segmental \\
\hline & GhCIPK16 & GhCIPK30 & 0.089 & 0.5674 & 0.1569 & Yes & Segmental \\
\hline & GhCIPK16 & GhCIPK55 & 0 & 0.0303 & 0 & No & Segmental \\
\hline & GhCIPK16 & GhCIPK68 & 0.089 & 0.4512 & 0.1973 & Yes & Segmental \\
\hline & GhCIPK18 & GhCIPK56 & 0.0082 & 0 & 0 & No & Segmental \\
\hline & GhCIPK19 & GhCIPK25 & 0.092 & 0.4042 & 0.2276 & Yes & Segmental \\
\hline & GhCIPK19 & GhCIPK57 & 0.016 & 0 & 0 & No & Segmental \\
\hline & GhCIPK19 & GhCIPK64 & 0.101 & 0.4042 & 0.2499 & Yes & Segmental \\
\hline & GhCIPK2 & GhCIPK39 & 0.008 & 0 & 0 & No & Segmental \\
\hline & GhCIPK20 & GhCIPK59 & 0.0081 & 0.0606 & 0.1337 & Yes & Segmental \\
\hline & GhCIPK20 & GhCIPK74 & 0.0765 & 0.7726 & 0.099 & Yes & Segmental \\
\hline & GhCIPK20 & GhCIPK43 & 0.0812 & 0.5899 & 0.1377 & Yes & Segmental \\
\hline & GhCIPK20 & GhCIPK52 & 0.0905 & 0.7899 & 0.1146 & Yes & Segmental \\
\hline & GhCIPK20 & GhCIPK37 & 0.0676 & 0.8566 & 0.0789 & Yes & Segmental \\
\hline & GhCIPK21 & GhCIPK44 & 0.1332 & 0.742 & 0.1795 & Yes & Segmental \\
\hline & GhCIPK21 & GhCIPK53 & 0.1358 & 0.7711 & 0.1761 & Yes & Segmental \\
\hline & GhCIPK21 & GhCIPK60 & 0 & 0.0289 & 0 & No & Segmental \\
\hline & GhCIPK22 & GhCIPK28 & 0.0673 & 0.3641 & 0.1848 & Yes & Segmental \\
\hline & GhCIPK22 & GhCIPK45 & 0.0495 & 0.4803 & 0.1031 & Yes & Segmental \\
\hline & GhCIPK22 & GhCIPK61 & 0.0081 & 0.0296 & 0.2736 & Yes & Segmental \\
\hline & GhCIPK22 & GhCIPK66 & 0.0585 & 0.4125 & 0.1418 & Yes & Segmental \\
\hline & GhCIPK23 & GhCIPK35 & 0.1488 & 0.405 & 0.3674 & Yes & Segmental \\
\hline & GhCIPK23 & GhCIPK62 & 0 & 0.0284 & 0 & No & Segmental \\
\hline & GhCIPK23 & GhCIPK73 & 0.1488 & 0.3597 & 0.4137 & Yes & Segmental \\
\hline & GhCIPK24 & GhCIPK63 & 0.0083 & 0.0541 & 0.1534 & Yes & Segmental \\
\hline & GhCIPK25 & GhCIPK57 & 0.0927 & 0.3908 & 0.2372 & Yes & Segmental \\
\hline & GhCIPK25 & GhCIPK64 & 0.024 & 0 & 0 & No & Segmental \\
\hline & GhCIPK26 & GhCIPK80 & 0.0159 & 0.1762 & 0.0902 & Yes & Segmental \\
\hline & GhCIPK27 & GhCIPK65 & 0 & 0.0586 & 0 & No & Segmental \\
\hline & GhCIPK28 & GhCIPK45 & 0.058 & 0.611 & 0.0949 & Yes & Segmental \\
\hline & GhCIPK28 & GhCIPK61 & 0.0757 & 0.422 & 0.1794 & Yes & Segmental \\
\hline & GhCIPK28 & GhCIPK66 & 0.0081 & 0.0929 & 0.0872 & Yes & Segmental \\
\hline & GhCIPK29 & GhCIPK67 & 0.0158 & 0 & 0 & No & Segmental \\
\hline & GhCIPK3 & GhCIPK32 & 0.6002 & 3.0016 & 0.2 & Yes & Segmental \\
\hline & GhCIPK3 & GhCIPK40 & 0.5373 & 0 & 0 & No & Segmental \\
\hline & GhCIPK3 & GhCIPK71 & 0.6244 & 2.6547 & 0.2352 & Yes & Segmental \\
\hline & GhCIPK30 & GhCIPK47 & 0.1077 & 0.7646 & 0.1409 & Yes & Segmental \\
\hline & GhCIPK30 & GhCIPK55 & 0.089 & 0.633 & 0.1406 & Yes & Segmental \\
\hline & GhCIPK30 & GhCIPK68 & 0 & 0.0609 & 0 & No & Segmental \\
\hline & GhCIPK31 & GhCIPK69 & 0 & 0.0296 & 0 & No & Segmental \\
\hline & GhCIPK32 & GhCIPK40 & 0.1707 & 0.3898 & 0.4379 & Yes & Segmental \\
\hline & GhCIPK32 & GhCIPK71 & 0.0078 & 0 & 0 & No & Segmental \\
\hline
\end{tabular}


Table 1. Cont.

\begin{tabular}{|c|c|c|c|c|c|c|c|}
\hline Species & $\begin{array}{c}\text { Duplicated } \\
\text { Gene } 1\end{array}$ & $\begin{array}{l}\text { Duplicated } \\
\text { Gene } 2\end{array}$ & $\mathbf{K a}$ & Ks & $\mathrm{Ka} / \mathrm{Ks}$ & $\begin{array}{l}\text { Purifying } \\
\text { Selection }\end{array}$ & $\begin{array}{c}\text { Duplicate } \\
\text { Type }\end{array}$ \\
\hline & GhCIPK33 & GhCIPK70 & 0.0081 & 0.0291 & 0.2784 & Yes & Segmental \\
\hline & GhCIPK34 & GhCIPK72 & 0.0166 & 0.0564 & 0.2943 & Yes & Segmental \\
\hline & GhCIPK35 & GhCIPK62 & 0.1488 & 0.4532 & 0.3283 & Yes & Segmental \\
\hline & GhCIPK35 & GhCIPK73 & 0.0167 & 0.027 & 0.6185 & Yes & Segmental \\
\hline & GhCIPK37 & GhCIPK43 & 0.0638 & 0.5677 & 0.1124 & Yes & Segmental \\
\hline & GhCIPK37 & GhCIPK52 & 0.0959 & 0.7194 & 0.1333 & Yes & Segmental \\
\hline & GhCIPK37 & GhCIPK59 & 0.0765 & 0.8566 & 0.0893 & Yes & Segmental \\
\hline & GhCIPK37 & GhCIPK74 & 0.0082 & 0.0574 & 0.1429 & Yes & Segmental \\
\hline & GhCIPK4 & GhCIPK42 & 0.0082 & 0 & 0 & No & Segmental \\
\hline & GhCIPK40 & GhCIPK71 & 0.1604 & 0.3953 & 0.4058 & Yes & Segmental \\
\hline & GhCIPK43 & GhCIPK52 & 0.0822 & 0.9098 & 0.0903 & Yes & Segmental \\
\hline & GhCIPK43 & GhCIPK59 & 0.0902 & 0.4754 & 0.1897 & Yes & Segmental \\
\hline & GhCIPK43 & GhCIPK74 & 0.0728 & 0.5677 & 0.1282 & Yes & Segmental \\
\hline & GhCIPK44 & GhCIPK53 & 0.1271 & 0.6696 & 0.1898 & Yes & Segmental \\
\hline & GhCIPK44 & GhCIPK60 & 0.1331 & 0.6735 & 0.1976 & Yes & Segmental \\
\hline & GhCIPK45 & GhCIPK61 & 0.0409 & 0.5525 & 0.074 & Yes & Segmental \\
\hline & GhCIPK45 & GhCIPK66 & 0.0494 & 0.6816 & 0.0725 & Yes & Segmental \\
\hline & GhCIPK47 & GhCIPK68 & 0.1077 & 0.7646 & 0.1409 & Yes & Segmental \\
\hline & GhCIPK48 & GhCIPK50 & 0.0418 & 0.1206 & 0.3466 & Yes & Segmental \\
\hline & GhCIPK48 & GhCIPK75 & 0 & 0.0574 & 0 & No & Segmental \\
\hline & GhCIPK48 & GhCIPK76 & 0.0418 & 0.189 & 0.2212 & Yes & Segmental \\
\hline & GhCIPK49 & GhCIPK72 & 0.1336 & 0.4847 & 0.2756 & Yes & Segmental \\
\hline & GhCIPK5 & GhCIPK41 & 0.0161 & 0.0305 & 0.5279 & Yes & Segmental \\
\hline & GhCIPK50 & GhCIPK75 & 0.0418 & 0.1206 & 0.3466 & Yes & Segmental \\
\hline & GhCIPK50 & GhCIPK76 & 0 & 0.0574 & 0 & No & Segmental \\
\hline & GhCIPK52 & GhCIPK59 & 0.0997 & 0.7138 & 0.1397 & Yes & Segmental \\
\hline & GhCIPK52 & GhCIPK68 & 0.3312 & 2.0206 & 0.1639 & Yes & Segmental \\
\hline & GhCIPK55 & GhCIPK74 & 0.3463 & 1.4874 & 0.2328 & Yes & Segmental \\
\hline & GhCIPK57 & GhCIPK64 & 0.1017 & 0.3908 & 0.2602 & Yes & Segmental \\
\hline & GhCIPK58 & GhCIPK77 & 0.0246 & 0.0908 & 0.2709 & Yes & Segmental \\
\hline & GhCIPK59 & GhCIPK74 & 0.0855 & 0.7726 & 0.1107 & Yes & Segmental \\
\hline & GhCIPK6 & GhCIPK13 & 0.0822 & 0.9098 & 0.0903 & Yes & Segmental \\
\hline & GhCIPK6 & GhCIPK20 & 0.0812 & 0.5899 & 0.1377 & Yes & Segmental \\
\hline & GhCIPK6 & GhCIPK37 & 0.0638 & 0.5677 & 0.1124 & Yes & Segmental \\
\hline & GhCIPK6 & GhCIPK43 & 0 & 0 & 0 & No & Segmental \\
\hline & GhCIPK6 & GhCIPK52 & 0.0822 & 0.9098 & 0.0903 & Yes & Segmental \\
\hline & GhCIPK6 & GhCIPK59 & 0.0902 & 0.4754 & 0.1897 & Yes & Segmental \\
\hline & GhCIPK6 & GhCIPK74 & 0.0728 & 0.5677 & 0.1282 & Yes & Segmental \\
\hline & GhCIPK61 & GhCIPK66 & 0.0669 & 0.4754 & 0.1407 & Yes & Segmental \\
\hline & GhCIPK62 & GhCIPK73 & 0.1488 & 0.405 & 0.3674 & Yes & Segmental \\
\hline & GhCIPK7 & GhCIPK21 & 0.1424 & 0.6837 & 0.2083 & Yes & Segmental \\
\hline & GhCIPK7 & GhCIPK44 & 0.0082 & 0.028 & 0.2929 & Yes & Segmental \\
\hline & GhCIPK7 & GhCIPK60 & 0.1423 & 0.76 & 0.1872 & Yes & Segmental \\
\hline & GhCIPK75 & GhCIPK76 & 0.0418 & 0.154 & 0.2714 & Yes & Segmental \\
\hline & GhCIPK78 & GhCIPK79 & 0 & 0 & 0 & No & Segmental \\
\hline & GhCIPK8 & GhCIPK22 & 0.0495 & 0.4803 & 0.1031 & Yes & Segmental \\
\hline & GhCIPK8 & GhCIPK28 & 0.058 & 0.611 & 0.0949 & Yes & Segmental \\
\hline & GhCIPK8 & GhCIPK45 & 0 & 0 & 0 & No & Segmental \\
\hline & GhCIPK8 & GhCIPK61 & 0.0409 & 0.5525 & 0.074 & Yes & Segmental \\
\hline & GhCIPK8 & GhCIPK66 & 0.0494 & 0.6816 & 0.0725 & Yes & Segmental \\
\hline & GhCIPK9 & GhCIPK46 & 0.0329 & 0.0293 & 1.1229 & No & Segmental \\
\hline \multirow[t]{7}{*}{ G. barbadense } & GbCIPK1 & GbCIPK37 & 0.0113 & 0.0325 & 0.3477 & Yes & Segmental \\
\hline & GbCIPK2 & GbCIPK38 & 0.0228 & 0.0581 & 0.3924 & Yes & Segmental \\
\hline & GbCIPK4 & GbCIPK41 & 0.0069 & 0.0391 & 0.1765 & Yes & Segmental \\
\hline & GbCIPK5 & GbCIPK40 & 0.0113 & 0.0165 & 0.6848 & Yes & Segmental \\
\hline & GbCIPK6 & GbCIPK14 & 0.13 & 0.4949 & 0.2627 & Yes & Segmental \\
\hline & GbCIPK6 & GbCIPK36 & 0.104 & 0.435 & 0.2391 & Yes & Segmental \\
\hline & GbCIPK6 & GbCIPK42 & 0.0068 & 0.0241 & 0.2822 & Yes & Segmental \\
\hline
\end{tabular}


Table 1. Cont.

\begin{tabular}{|c|c|c|c|c|c|c|c|}
\hline Species & $\begin{array}{l}\text { Duplicated } \\
\text { Gene } 1\end{array}$ & $\begin{array}{l}\text { Duplicated } \\
\text { Gene } 2\end{array}$ & Ка & Ks & $\mathrm{Ka} / \mathrm{Ks}$ & $\begin{array}{l}\text { Purifying } \\
\text { Selection }\end{array}$ & $\begin{array}{c}\text { Duplicate } \\
\text { Type }\end{array}$ \\
\hline & GbCIPK6 & GbCIPK51 & 0.13 & 0.5103 & 0.2548 & Yes & Segmental \\
\hline & GbCIPK6 & GbCIPK59 & 0.0948 & 0.571 & 0.166 & Yes & Segmental \\
\hline & GbCIPK6 & GbCIPK76 & 0.1015 & 0.4327 & 0.2346 & Yes & Segmental \\
\hline & GbCIPK6 & GbCIPK78 & 0.0974 & 0.5699 & 0.1709 & Yes & Segmental \\
\hline & GbCIPK7 & GbCIPK21 & 0.118 & 0.3925 & 0.3006 & Yes & Segmental \\
\hline & GbCIPK7 & GbCIPK43 & 0.0134 & 0.0258 & 0.5194 & Yes & Segmental \\
\hline & GbCIPK7 & GbCIPK60 & 0.1231 & 0.3939 & 0.3125 & Yes & Segmental \\
\hline & GbCIPK8 & GbCIPK22 & 0.0663 & 0.2358 & 0.2812 & Yes & Segmental \\
\hline & GbCIPK8 & GbCIPK44 & 0.0114 & 0.0078 & 1.4615 & No & Segmental \\
\hline & GbCIPK8 & GbCIPK61 & 0.0665 & 0.2545 & 0.2613 & Yes & Segmental \\
\hline & GbCIPK8 & GbCIPK67 & 0.0904 & 0.378 & 0.2392 & Yes & Segmental \\
\hline & GbCIPK9 & GbCIPK45 & 0.0113 & 0.0588 & 0.1922 & Yes & Segmental \\
\hline & GbCIPK10 & GbCIPK16 & 0.1053 & 0.3484 & 0.3022 & Yes & Segmental \\
\hline & GbCIPK10 & GbCIPK30 & 0.1323 & 0.477 & 0.2774 & Yes & Segmental \\
\hline & GbCIPK10 & GbCIPK46 & 0.009 & 0.0083 & 1.0843 & No & Segmental \\
\hline & GbCIPK10 & GbCIPK70 & 0.1322 & 0.4774 & 0.2769 & Yes & Segmental \\
\hline & GbCIPK11 & GbCIPK47 & 0.0023 & 0.0241 & 0.0954 & Yes & Segmental \\
\hline & GbCIPK11 & GbCIPK49 & 0.0442 & 0.2282 & 0.1937 & Yes & Segmental \\
\hline & GbCIPK11 & GbCIPK77 & 0.0466 & 0.2171 & 0.2146 & Yes & Segmental \\
\hline & GbCIPK12 & GbCIPK34 & 0.0948 & 0.3398 & 0.279 & Yes & Segmental \\
\hline & GbCIPK12 & GbCIPK 48 & 0.0068 & 0.0161 & 0.4224 & Yes & Segmental \\
\hline & GbCIPK12 & GbCIPK74 & 0.0922 & 0.3275 & 0.2815 & Yes & Segmental \\
\hline & GbCIPK13 & GbCIPK50 & 0.0045 & 0 & 0 & No & Segmental \\
\hline & GbCIPK14 & GbCIPK36 & 0.1155 & 0.4284 & 0.2696 & Yes & Segmental \\
\hline & GbCIPK14 & GbCIPK42 & 0.1325 & 0.4982 & 0.266 & Yes & Segmental \\
\hline & GbCIPK14 & GbCIPK51 & 0 & 0.0158 & 0 & No & Segmental \\
\hline & GbCIPK14 & GbCIPK59 & 0.1209 & 0.5682 & 0.2128 & Yes & Segmental \\
\hline & GbCIPK14 & GbCIPK76 & 0.1103 & 0.4125 & 0.2674 & Yes & Segmental \\
\hline & GbCIPK14 & GbCIPK78 & 0.1263 & 0.5671 & 0.2227 & Yes & Segmental \\
\hline & GbCIPK15 & GbCIPK52 & 0.0067 & 0.0418 & 0.1603 & Yes & Segmental \\
\hline & GbCIPK16 & GbCIPK30 & 0.0985 & 0.5022 & 0.1961 & Yes & Segmental \\
\hline & GbCIPK16 & GbCIPK54 & 0.0067 & 0.0248 & 0.2702 & Yes & Segmental \\
\hline & GbCIPK16 & GbCIPK70 & 0.0933 & 0.5184 & 0.18 & Yes & Segmental \\
\hline & GbCIPK17 & GbCIPK27 & 0.0799 & 0.2076 & 0.3849 & Yes & Segmental \\
\hline & GbCIPK17 & GbCIPK55 & 0.0022 & 0.0257 & 0.0856 & Yes & Segmental \\
\hline & GbCIPK17 & GbCIPK66 & 0.0762 & 0.2021 & 0.377 & Yes & Segmental \\
\hline & GbCIPK18 & GbCIPK56 & 0.0022 & 0.1048 & 0.021 & Yes & Segmental \\
\hline & GbCIPK19 & GbCIPK57 & 0.0114 & 0.0656 & 0.1738 & Yes & Segmental \\
\hline & GbCIPK20 & GbCIPK58 & 0.0249 & 0.0339 & 0.7345 & Yes & Segmental \\
\hline & GbCIPK21 & GbCIPK43 & 0.1207 & 0.404 & 0.2988 & Yes & Segmental \\
\hline & GbCIPK21 & GbCIPK52 & 0.1214 & 0.4081 & 0.2975 & Yes & Segmental \\
\hline & GbCIPK21 & GbCIPK60 & 0.009 & 0.0163 & 0.5521 & Yes & Segmental \\
\hline & GbCIPK22 & GbCIPK44 & 0.4258 & 1.6158 & 0.2635 & Yes & Segmental \\
\hline & GbCIPK22 & GbCIPK61 & 0.0045 & 0.0317 & 0.142 & Yes & Segmental \\
\hline & GbCIPK22 & GbCIPK67 & 0.0953 & 0.3812 & 0.25 & Yes & Segmental \\
\hline & GbCIPK23 & GbCIPK35 & 0.0848 & 0.3013 & 0.2814 & Yes & Segmental \\
\hline & GbCIPK23 & GbCIPK62 & 0.0045 & 0.0082 & 0.5488 & Yes & Segmental \\
\hline & GbCIPK23 & GbCIPK75 & 0.0823 & 0.3137 & 0.2624 & Yes & Segmental \\
\hline & GbCIPK24 & GbCIPK63 & 0.009 & 0.0586 & 0.1536 & Yes & Segmental \\
\hline & GbCIPK25 & GbCIPK57 & 0.0812 & 0.3393 & 0.2393 & Yes & Segmental \\
\hline & GbCIPK25 & GbCIPK64 & 0.0068 & 0.0078 & 0.8718 & Yes & Segmental \\
\hline & GbCIPK26 & GbCIPK65 & 0.025 & 0.0593 & 0.4216 & Yes & Segmental \\
\hline & GbCIPK27 & GbCIPK55 & 0.0811 & 0.1914 & 0.4237 & Yes & Segmental \\
\hline & GbCIPK27 & GbCIPK66 & 0.0022 & 0.0081 & 0.2716 & Yes & Segmental \\
\hline & GbCIPK27 & GbCIPK71 & 0.1188 & 0.2344 & 0.5068 & Yes & Segmental \\
\hline & GbCIPK28 & GbCIPK68 & 0.0046 & 0.0076 & 0.6053 & Yes & Segmental \\
\hline & GbCIPK29 & GbCIPK69 & 0.0228 & 0.0245 & 0.9306 & Yes & Segmental \\
\hline & GbCIPK3 & GbCIPK32 & 0.1067 & 0.433 & 0.2464 & Yes & Segmental \\
\hline
\end{tabular}


Table 1. Cont.

\begin{tabular}{|c|c|c|c|c|c|c|c|}
\hline Species & $\begin{array}{c}\text { Duplicated } \\
\text { Gene } 1\end{array}$ & $\begin{array}{l}\text { Duplicated } \\
\text { Gene } 2\end{array}$ & Ка & Ks & $\mathrm{Ka} / \mathrm{Ks}$ & $\begin{array}{l}\text { Purifying } \\
\text { Selection }\end{array}$ & $\begin{array}{c}\text { Duplicate } \\
\text { Type }\end{array}$ \\
\hline & GbCIPK3 & GbCIPK39 & 0.0136 & 0.0409 & 0.3325 & Yes & Segmental \\
\hline & GbCIPK3 & GbCIPK73 & 0.099 & 0.4178 & 0.237 & Yes & Segmental \\
\hline & GbCIPK30 & GbCIPK46 & 0.1268 & 0.4942 & 0.2566 & Yes & Segmental \\
\hline & GbCIPK30 & GbCIPK54 & 0.1062 & 0.5344 & 0.1987 & Yes & Segmental \\
\hline & GbCIPK30 & GbCIPK70 & 0.0068 & 0.0238 & 0.2857 & Yes & Segmental \\
\hline & GbCIPK31 & GbCIPK71 & 0.0045 & 0.0081 & 0.5556 & Yes & Segmental \\
\hline & GbCIPK32 & GbCIPK72 & 0.4802 & 1.8172 & 0.2643 & Yes & Segmental \\
\hline & GbCIPK33 & GbCIPK73 & 0.0114 & 0.0315 & 0.3619 & Yes & Segmental \\
\hline & GbCIPK34 & GbCIPK 48 & 0.0998 & 0.3671 & 0.2719 & Yes & Segmental \\
\hline & GbCIPK34 & GbCIPK74 & 0.0091 & 0.0406 & 0.2241 & Yes & Segmental \\
\hline & GbCIPK35 & GbCIPK62 & 0.0848 & 0.3013 & 0.2814 & Yes & Segmental \\
\hline & GbCIPK35 & GbCIPK75 & 0.0067 & 0.0257 & 0.2607 & Yes & Segmental \\
\hline & GbCIPK36 & GbCIPK42 & 0.1065 & 0.4237 & 0.2514 & Yes & Segmental \\
\hline & GbCIPK36 & GbCIPK51 & 0.1155 & 0.4284 & 0.2696 & Yes & Segmental \\
\hline & GbCIPK36 & GbCIPK59 & 0.1143 & 0.4103 & 0.2786 & Yes & Segmental \\
\hline & GbCIPK36 & GbCIPK76 & 0.0183 & 0.0158 & 1.1582 & No & Segmental \\
\hline & GbCIPK36 & GbCIPK78 & 0.1222 & 0.4095 & 0.2984 & Yes & Segmental \\
\hline & GbCIPK39 & GbCIPK73 & 0.1041 & 0.4619 & 0.2254 & Yes & Segmental \\
\hline & GbCIPK42 & GbCIPK51 & 0.4678 & 1.2446 & 0.3759 & Yes & Segmental \\
\hline & GbCIPK42 & GbCIPK59 & 0.4547 & 1.5066 & 0.3018 & Yes & Segmental \\
\hline & GbCIPK42 & GbCIPK76 & 0.4425 & 1.2039 & 0.3676 & Yes & Segmental \\
\hline & GbCIPK42 & GbCIPK78 & 0.4591 & 1.5003 & 0.306 & Yes & Segmental \\
\hline & GbCIPK43 & GbCIPK60 & 0.1206 & 0.4054 & 0.2975 & Yes & Segmental \\
\hline & GbCIPK44 & GbCIPK61 & 0.0639 & 0.2674 & 0.239 & Yes & Segmental \\
\hline & GbCIPK44 & GbCIPK67 & 0.0928 & 0.3942 & 0.2354 & Yes & Segmental \\
\hline & GbCIPK46 & GbCIPK70 & 0.1268 & 0.4946 & 0.2564 & Yes & Segmental \\
\hline & GbCIPK47 & GbCIPK49 & 0.0418 & 0.1971 & 0.2121 & Yes & Segmental \\
\hline & GbCIPK47 & GbCIPK77 & 0.0442 & 0.1865 & 0.237 & Yes & Segmental \\
\hline & GbCIPK48 & GbCIPK74 & 0.0972 & 0.3542 & 0.2744 & Yes & Segmental \\
\hline & GbCIPK49 & GbCIPK77 & 0.0045 & 0.0158 & 0.2848 & Yes & Segmental \\
\hline & GbCIPK51 & GbCIPK59 & 0.1209 & 0.5516 & 0.2192 & Yes & Segmental \\
\hline & GbCIPK51 & GbCIPK76 & 0.1103 & 0.4125 & 0.2674 & Yes & Segmental \\
\hline & GbCIPK51 & GbCIPK78 & 0.1263 & 0.5505 & 0.2294 & Yes & Segmental \\
\hline & GbCIPK54 & GbCIPK70 & 0.101 & 0.5514 & 0.1832 & Yes & Segmental \\
\hline & GbCIPK55 & GbCIPK66 & 0.0786 & 0.1809 & 0.4345 & Yes & Segmental \\
\hline & GbCIPK57 & GbCIPK64 & 0.0761 & 0.354 & 0.215 & Yes & Segmental \\
\hline & GbCIPK59 & GbCIPK78 & 0.0113 & 0 & 0 & No & Segmental \\
\hline & GbCIPK59 & GbCIPK76 & 0.1092 & 0.4081 & 0.2676 & Yes & Segmental \\
\hline & GbCIPK61 & GbCIPK67 & 0.0905 & 0.4023 & 0.225 & Yes & Segmental \\
\hline & GbCIPK62 & GbCIPK75 & 0.0823 & 0.3137 & 0.2624 & Yes & Segmental \\
\hline & GbCIPK66 & GbCIPK71 & 0.1162 & 0.2235 & 0.5199 & Yes & Segmental \\
\hline & GbCIPK76 & GbCIPK78 & 0.1171 & 0.4074 & 0.2874 & Yes & Segmental \\
\hline
\end{tabular}

2.4. Analysis of Cis-Elements and Prediction of Transcription Factor Binding Sites in the Promoter Regions of GhCIPKs

Analysis of cis-elements in genes could provide critical evidence for the gene's function. Many studies reported that CIPK genes play key roles in various stresses [17,22,25]. Here, the putative stress-related and hormone-related cis-elements were scanned in the $1.5 \mathrm{~kb}$ upstream of the start codons of 80 GhCIPKs using the PlantCARE database. In total, 12 stress-related and hormone-related cis-elements were predicted in the promoters of 80 GhCIPKs (Table S8). Among those GhCIPKs, 36 GhCIPKs had the abscisic acid (ABA) responsive element (ABRE), 8 GhCIPKs harbored the auxin responsive element (TGA-element and AuxRR-core), 17 GhCIPKs contained defense and stress responsive elements (AT-rich and TC-rich repeats), 16 GhCIPKs contained the drought responsive 
element (MBS), 25 GhCIPKs harbored the gibberellin (GA) responsive element (TATC-box, P-box, and GARE-motif), 22 GhCIPKs had the salicylic acid (SA) responsive element (TCA-element and SARE), 81 GhCIPKs possessed the light responsive element (G-box, Box 4, GT1-motif, MRE, AE-box, ACE, Sp1 and I-box), 45 GhCIPKs had the methyl jasmonate (MeJA) responsive element (TGACG-motif and CGTCA-motif), 45 GhCIPKs contained the anaerobic induction responsive element (ARE), 19 GhCIPKs possessed the low temperature-responsive element (LTR), 25 GhCIPKs had the wound-responsive element (WUN-motif), and 15 GhCIPKs contained the wounding and pathogen responsive element (W box). In total, 68 cis-elements were related to ABA, 10 to auxin, 19 to defense and stress, 23 to drought, 33 to GA, 22 to SA, 341 to light, 66 to MeJA, 64 to anaerobic induction, 22 to low-temperature, 31 to wound-responsive, and 15 to wounding and pathogen were identified in the 80 GhCIPKs. These results suggest that GhCIPKs could be involved in various regulatory mechanisms when cotton plants are subjected to various stresses.

It is well known that TFs regulate the transcription of their target genes by binding certain upstream elements [39]. To explore the regulatory interactions between TFs and GhCIPKs, we further searched highly conserved transcription factor binding sites (TFBSs) in $1.5 \mathrm{~kb}$ promoter regions of the 80 GhCIPKs. The results showed that $166 \mathrm{TFs}$ belonging to 28 families might bind to the $1.5 \mathrm{~kb}$ promoter regions of 80 GhCIPKs (Table S9). Among the $28 \mathrm{TF}$ families, many were found in plant-specific families, such as the B3, G2-like, NAC, TCP, and LBD. However, many TFs were also found in animals, bacteria, and yeast, such as the ERF, C2H2, HD-ZIP, MYB and TALE families. Numerous reports emphasize that these TF families play key roles in plant responses to environmental stimuli and regulation, such as seed storage protein synthesis, carbohydrate metabolism, plant defense mechanisms, seed germination, hormonal signal transduction and response to various biotic and abiotic stresses [40-43].

\subsection{Expression Profiling of GhCIPK Genes in Different Tissues and Under Various Stresses}

To understand the possible functions of GhCIPK genes in different tissues, the gene expression patterns of 80 GhCIPK genes from the public RNA-seq data of TM-1 were analyzed in different tissues (root, stem, leaf, cotyledon, torus, petal, stamen, pistil, and calycle) and developmental stages (ovule and fiber) (Figure 4). As shown in Figure 4, 7 GhCIPKs were highly expressed in all tested tissues of TM-1, including GhCIPK4, GhCIPK6, GhCIPK13, GhCIPK39, GhCIPK41, GhCIPK42, and GhCIPK65; GhCIPK72 was expressed at low levels in all tested tissues except torus and pistil. This indicated that some GhCIPK genes had multiple biological functions during cotton development. Additionally, several GhCIPK genes were specifically expressed in one or several specific tissues. For example, GhCIPK58 was only expressed in petals and stamens. GhCIPK26 and GhCIPK80 were only expressed in stamens, while GhCIPK1, GhCIPK10, and GhCIPK47 were highly expressed in stamens than in other tissues. These results imply that these genes play key roles in the development or function of specific tissues.

Previous studies reported that CIPK genes could play important roles in plant responses to various stresses $[14,22,25,44]$. Therefore, we analyzed the expression patterns of the 80 GhCIPKs under various stresses, including cold, hot, salt, and drought stresses, using the published RNA-seq data of TM-1. As shown in Figure 5, the relative expression levels of some GhCIPKs were either induced or suppressed by these four treatments. There were 16 GhCIPKs downregulated under the four stress treatments. The expression levels of GhCIPK3 and GhCIPK53 were significantly upregulated under salt or drought stress. The expression of GhCIPK11 was significantly upregulated under cold stress, and GhCIPK37 was upregulated under drought stress. Several GhCIPKs were upregulated at only one time point in at least one treatment. For example, GhCIPK13 was upregulated at $3 \mathrm{~h}$ under drought stress, while GhCIPK48 and GhCIPK71 were upregulated at $12 \mathrm{~h}$ under hot, drought, or cold stress. 


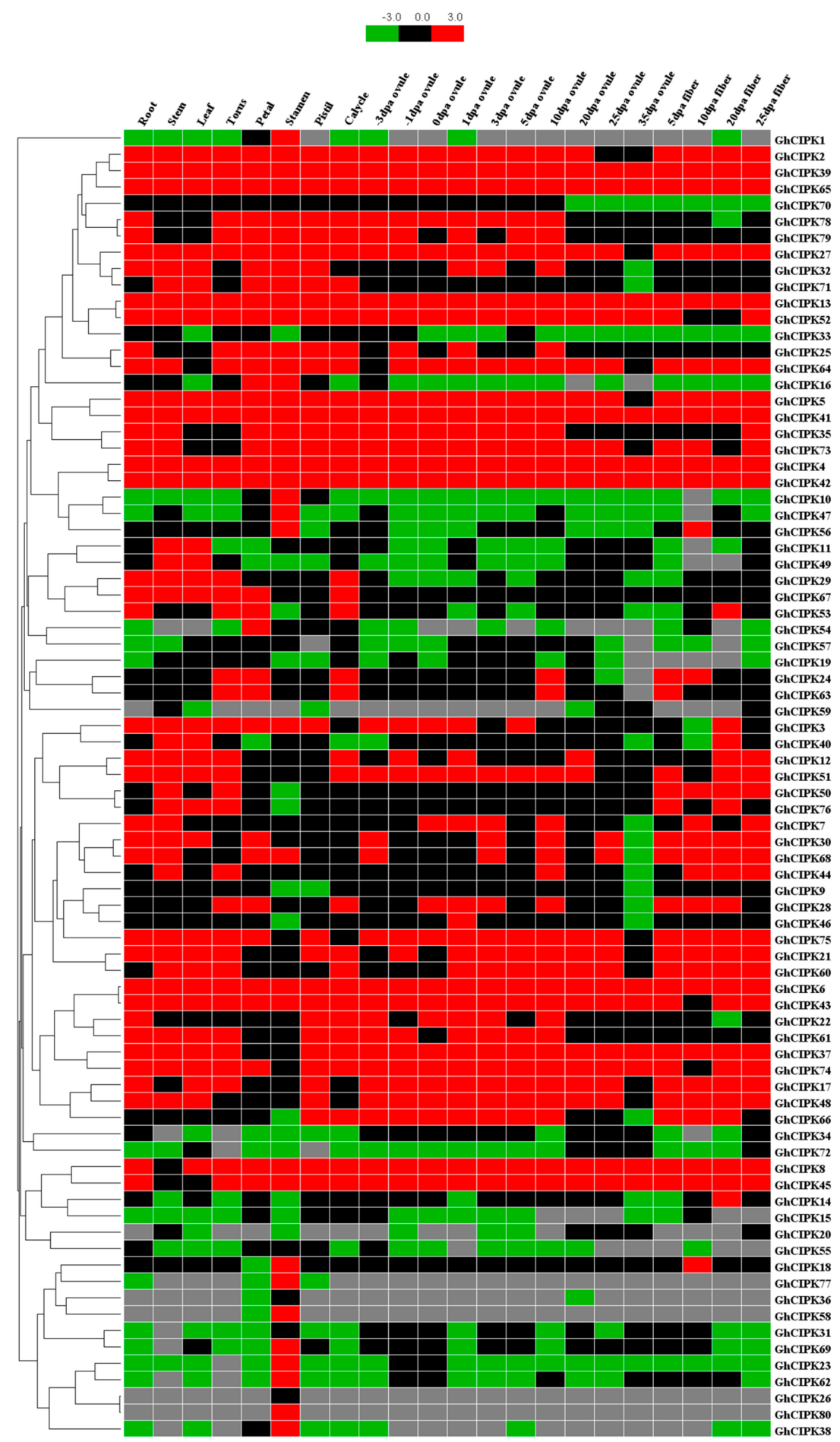

Figure 4. Expression patterns of 80 ChCIPK genes in different tissues of TM-1. The raw data for RNA-Seq of G. hirsutum acc. TM-1 [34] was downloaded and used to analyze the expression patterns of ChCIPK genes. The color bar represents the expression values in log2 of fragments per kilobase per million reads (FPKMs). The gray color represents that there is no expression. 


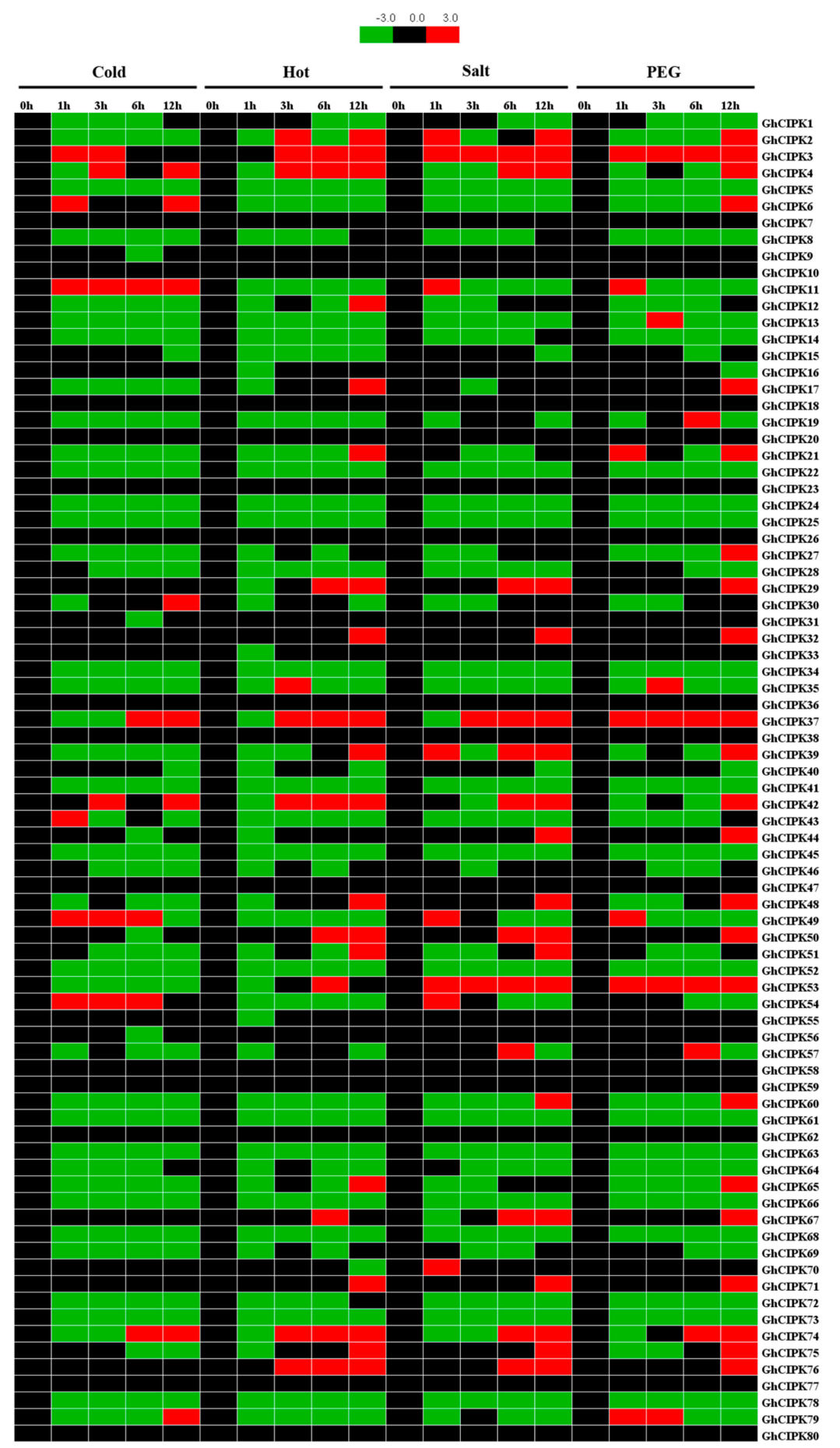

Figure 5. Expression patterns of 80 ChCIPK genes in response to various stresses. The colored bar represents the relative expression levels.

To further confirm the expression patterns of GhCIPK genes in response to environmental stresses, we selected four GhCIPK genes to examine their expression profiles under salt stress and cold stress. As shown in Figure 6, GhCIPK53 and GhCIPK74 showed similar expression patterns, which were upregulated by different salt treatments. The expression level of GhCIPK11 was only upregulated at $150 \mathrm{mM}$ salt treatment, while GhCIPK37 was upregulated at $150 \mathrm{mM}$ and $200 \mathrm{mM}$ salt treatments, respectively. Under cold stress, three genes (GhCIPK11, GhCIPK37, and GhCIPK74) were significantly upregulated; the expression level of GhCIPK53 quickly peaked after $12 \mathrm{~h}$ and then decreased at $24 \mathrm{~h}$ 
of cold stress. These results suggest that GhCIPK genes might enhance the adaptability of cotton to various abiotic stresses.
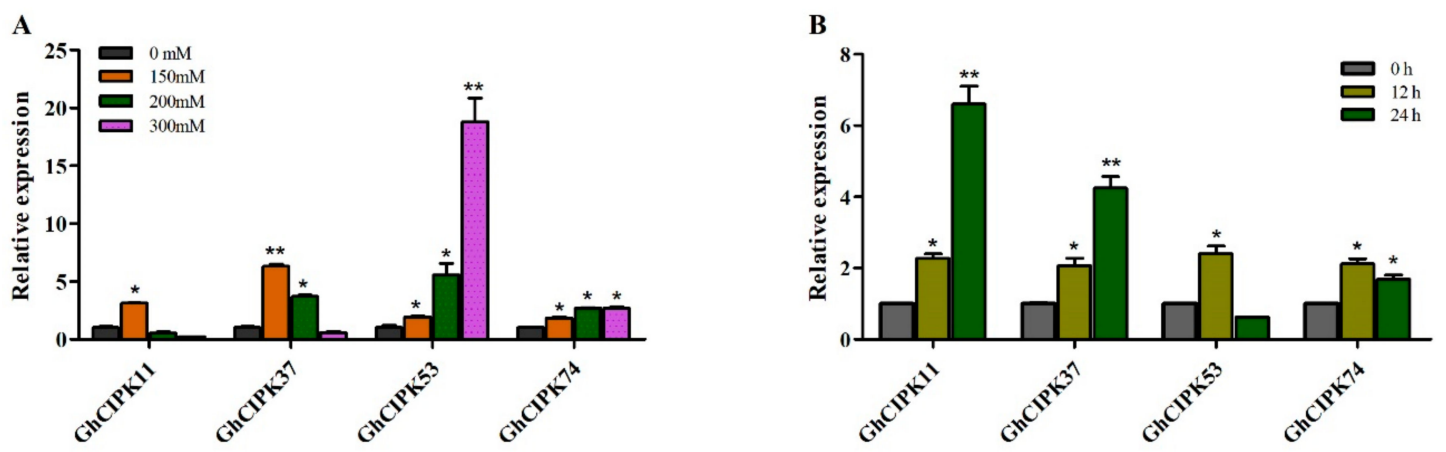

Figure 6. Relative expression levels of four ChCIPK genes under salt and cold treatments. (A) Relative expression levels of four ChCIPK genes under salt treatment; (B) relative expression levels of four ChCIPK genes under cold treatment. The GhUBQ7 was used as an internal control. Error bars indicate the standard deviations of three independent experiments. Significant differences are determined by $t$-test compared to control treatment $\left({ }^{*} p<0.05 ;{ }^{* *} p<0.01\right)$.

\subsection{Co-Localization and Sequence Variation of GhCIPK Genes with QTLs for Seed Oil and Protein Content}

Although there are many studies in genetic analysis and QTL mapping of oil content in different cotton populations [26,28], the genetic relationships of cotton CIPK gene family with oil and protein content has not been reported on cotton. Here, we co-localized the GhCIPK genes with reported quantitative trail loci (QTLs) for oil and protein contents. The QTLs of intraspecific G. hirsutum and interspecific G. hirsutum $\times$ G. barbadense populations were downloaded from the CottonQTL database [45,46] and a recently published article [47]. Finally, 14 GhCIPK genes were mapped with the anchored cotton oil QTL or protein QTLs within a $20 \mathrm{cM}$ region (Figure S8). There were two genes (GhCIPK4 and GhCIPK5) on chromosome A03/c3, two genes (GhCIPK13 and GhCIPK14) on A06/c6, and one gene (GhCIPK37) on A13/c13 located within oil or protein content QTLs in the At subgenome of G. hirsutum. Two genes (GhCIPK41 and GhCIPK42) on chromosome D03/c14, two genes (GhCIPK47 and GhCIPK48) on chromosome D05/c19, two genes (GhCIPK61 and GhCIPK62) on chromosome D07/c16, two genes (GhCIPK65 and GhCIPK66) on chromosome D09/c23, and one gene (GhCIPK74) on chromosome D13/c18 were located within the oil or protein QTLs in the Dt subgenome of G. hirsutum.

We further identified sequence variation of the 14 GhCIPKs by scanning the RNA-seq data of CRI 36 and Hai 7124. Finally, 17 SNPs, classified as non-synonymous, were identified from the seven GhCIPKs (Table S10). Additionally, these SNPs were further analyzed in the five published cotton genome data (TM-1_NAU, TM-1_ZJU, 3-79, Xinhai 21 and Hai 7124), and nine SNPs of four GhCIPKs were confirmed as non-synonymous mutations. Primers of the nine SNPs were designed (Table S11) and screened in a backcross inbred line (BIL) population of 180 lines derived from a backcross between CRI 36 and Hai 7124 as described previously [48]. Five SNPs from four GhCIPKs were found to be significantly associated with oil content in the BIL population (Table S12). For example, in the BIL population, GhCIPK14-1273 (T/G) and GhCIPK42-340 (T/C) resulted in a change from cysteine to glycine and from phenylalanine to leucine, respectively; the two SNPs were found to be significantly associated with oil content in 2016XJ (-0.197 and -0.182). GhCIPK47-1318 (A/C) and GhCIPK66-917 (G/A) resulted in lysine to glutamine and a glycine to glutamic acid, respectively. The two SNPs were also found to be significantly associated with oil content in 2016AY $(-0.162,-0.156)$. GhCIPK66-406 (T/G), which resulted in a tyrosine to aspartic acid change, was significantly associated with oil content in 2015AY $(-0.209)$. However, the five SNPs were only associated with oil content in one test, and the association between the five SNPs and oil content needs further studies. These results show that most GhCIPK genes were not associated with natural variations in cotton oil content. 
One recent report showed that the overexpression of $B n C I P K 9$ during seed development reduced seed oil content in transgenic B. napus, and the seed oil content of Atcipk9 mutants was significantly higher than that of WT plants [29]. To confirm whether GhCIPK genes affect cottonseed oil content, the plasmid of recombinant vector (pBI121-GhCIPK6) was injected into the ovary of '11-0516' by syringe, and putative transgenic cotton seeds of $\mathrm{T}_{0}$ were harvested and further grown. Southern blotting revealed that two transgenic cotton plants had two copies, while no hybridizing band was found in wild-type (11-0516) (data not shown). The relative expression levels of GhCIPK6 in the two $\mathrm{T}_{3}$ transgenic cotton lines were higher than that in wild-type plants (Figure 7A). To characterize the biological function of GhCIPK6 in oil synthesis, the oil content of mature seeds from the two transgenic lines and wild-type plants was detected using an NMI20-Analyst nuclear magnetic resonance spectrometer (Niumag, Shanghai, China). As shown in Figure 7B, the two transgenic lines with increased expression levels of GhCIPK6 had significantly lower oil content (25.44\% and $32.37 \%)$ than wild-type plants (33.58\%). Furthermore, the relative proportions of $\mathrm{C} 18: 1$ and $\mathrm{C} 18: 1+\mathrm{C} 18: 1 \mathrm{~d} 6$ from transgenic lines cottonseeds were significantly increased, while the relative proportion of C18:2 was significantly decreased, compared to wild-type plants (Figure 7C). These results indicated that GhCIPK6 may play a negative role in oil synthesis.

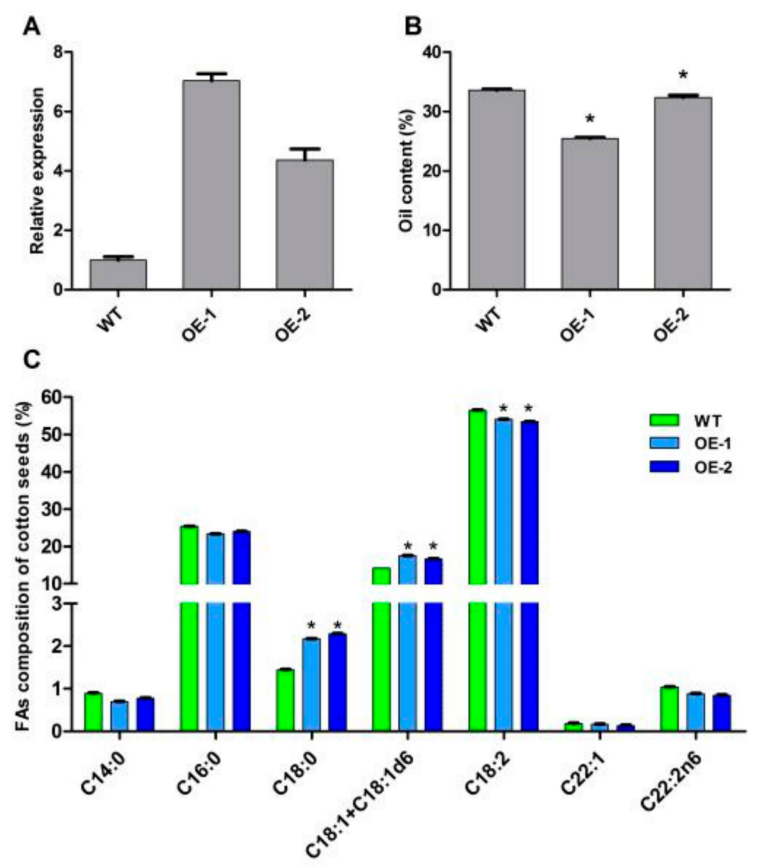

Figure 7. Overexpression of GhCIPK6 changed the oil content and fatty acid composition in transgenic cotton. (A) Real-time qRT-PCR analysis of GhCIPK6 expression in transgenic and wild type (WT) cotton plants. OE-1 and OE-2 were different transgenic lines; (B) cottonseed oil content of transgenic and WT cotton plants. Error bars indicate the standard error. Asterisks indicated significant differences $(p<0.05, *)$ between transgenic and WT plants; $(\mathbf{C})$ fatty acid composition in cottonseeds of transgenic and wild type (WT) plants. The student's t-tests was used to evaluate significant difference $\left(p<0.05,{ }^{*}\right)$.

\subsection{Predictions of Putative Molecular Regulatory Mechanisms of GhCIPKs}

The previous sections characterized the roles of GhCIPKs in different tissues, various stresses, and oil content. In this section, we characterize post-transcriptional regulation mediated by alternative splicing (AS) and miRNAs to predict the putative molecular regulatory mechanism of GhCIPKs in expression and their functional multiplicity.

AS, as a post-transcriptional regulation mechanism in eukaryotes, can generate multiple transcripts from the same gene to increase proteome diversity and regulate mRNA levels [49]. In Arabidopsis, it was estimated that more than $60 \%$ of exon-containing genes were subject to AS [50]. Many studies showed 
that more AS isoforms presented specific expression patterns in cells, tissues or different conditions, and the extent of AS was related to the complexity of tissues [51,52]. Based on our mRNA-seq data of cotton floral buds, the potential relationship between potential AS events of the GhCIPK genes and cotton floral buds was analyzed. We detected a total of 39 AS events from nine GhCIPKs in cotton floral buds, and seven GhCIPKs undergoing AS were associated with intron retention (IR) events, and the number of exon skipping (ES) (five GhCIPKs) was the second most common of AS events (Figure S9A). We randomly selected one gene (GhCIPK17) to validate the accuracy of the AS events using RT-PCR with the corresponding primers (Table S11). We found that each of the amplified fragment sizes was consistent with that of the predicted fragment (Figure S9B); subsequently, the amplified fragments were cloned for Sanger sequencing. Finally, the sequence consistency between cloned fragments and predicted sequences was observed based on our mRNA-seq of cotton floral buds.

In plants, miRNAs regulate gene expression at the post-transcriptional level by mediating mRNA cleavage or translational repression [53]. To explore the mechanisms of GhCIPK gene family regulated by miRNA, we searched the coding sequence regions of 80 GhCIPKs for putative target sites of cotton miRNA using the psRNATarget server with parameters described in Section 4. A total of 11 upland cotton miRNAs targeted 25 GhCIPKs (Figure 8, Table S13). As shown in Figure 8, GhCIPK3 and GhCIPK40 were both targeted by novel_mir_89 with sites in the kinase domain. GhCIPK13 was targeted by novel_mir_54 with sites in the 5'-end of CDS. GhCIPK16 and GhCIPK55 were both targeted by novel_mir_95 with sites in the kinase domain. One upland cotton novel_mir_32 regulated GhCIPK19, GhCIPK25, GhCIPK57, GhCIPK64, and GhCIPK78 in the kinase domain. In addition, we also found that GhCIPK6 and GhCIPK43 were both targeted by ghr-miR7498 with sites in the NAF domain. One upland cotton novel_mir_27 targeted GhCIPK26, GhCIPK48, GhCIPK75, and GhCIPK77 in the NAF domain. GhCIPK11 and GhCIPK49 were both targeted by ghr-miR7495a with sites in the kinase domain. One ghr-miR7509 targeted GhCIPK35 and GhCIPK73 in the NAF domain. Intriguingly, both novel_mir_95 and novel_mir_89 regulated GhCIPK3, but had different complementary sites and unrelated sequences. This case was also found in GhCIPK12, GhCIPK19, and GhCIPK40. For example, GhCIPK19 was targeted by ghr-miR7496a and novel_mir_32 with sites in the NAF domain and the kinase domain, respectively (Table S13). The divergence of miRNA target sites indicated that members of GhCIPKs might be regulated by different miRNAs. We further tested the expression patterns of the relationship between miRNAs and GhCIPK genes in cotton ovules through qRT-PCR analysis (Figure S10). Three ghr-miRNAs (novel_mir_54, ghr-miR7498, and novel_mir_27) and their target GhCIPK mRNAs (GhCIPK5, GhCIPK6, and GhCIPK58) showed negative regulatory relationships in cotton ovules. This implied that some cotton miRNAs may play important roles in cotton ovules by regulating these GhCIPK genes. 

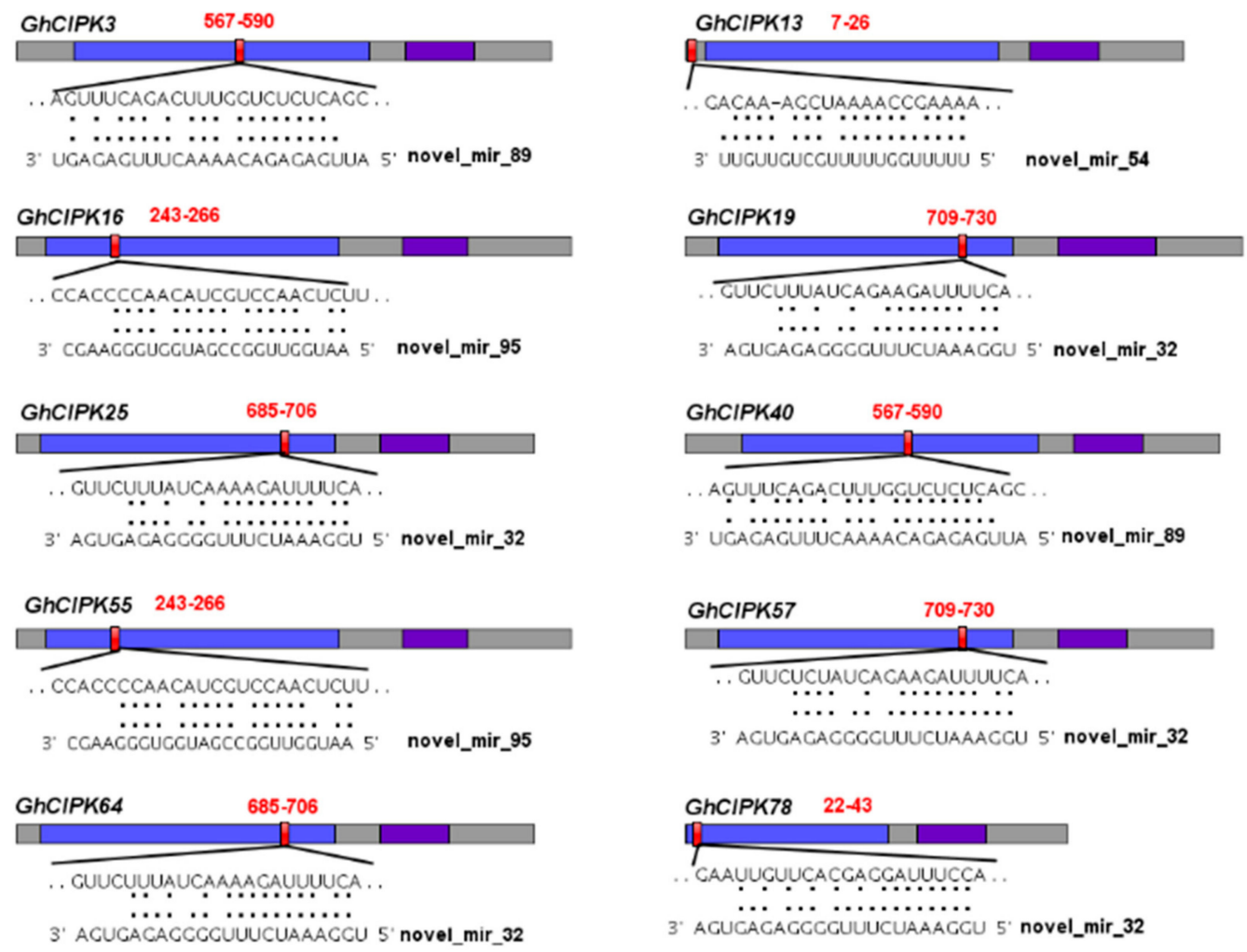

Figure 8. The miRNA-mediated targeting regulatory relationships of GhCIPKs. The ORFs (open reading frames) of GhCIPKs are indicated by heavy grey boxes. The protein kinase domain (PF00069) and the NAF domain (PF03822) are shown in blue and purple boxes, respectively. miRNA complementary sites with the nucleotide positions of CIPK genes are filled by the color red.

Nine GhCIPKs contained 34 AS events, including alternative $3^{\prime}$ acceptor sites (AA), alternative 5' donor sites (AD), intron retention (IR), and exon skipping (ES) were detected from mRNA-seq data in cotton floral buds, (Figure S9A). To understand how miRNAs interact with isoforms, we then predicted target sites for these cotton miRNAs using CIPK full-length isoforms. A total of nine isoforms from two GhCIPKs were identified to be potential targets of two novel miRNAs (novel_mir_27 targeted isoforms from GhCIPK48 and novel_mir_63 targeted isoforms from GhCIPK51) (Figure S11). Furthermore, we examined the effect of AS on gain/loss of miRNA target sites among the nine GhCIPK isoforms regulated by miRNAs; the results showed that the miRNA target sites in nine isoforms were not affected by AS events (Figure S11). This result is possibly because only one or two miRNAs regulated the isoforms of the GhCIPKs or only part of the isoforms transcribed from GhCIPK genes were detected from our mRNA-seq data. Similar phenomena could also be detected in the cotton CAT gene family in Figure S6 [39].

\section{Discussion}

\subsection{Phylogenetic Analysis and Evolution of CIPK Genes in Gossypium}

The CIPK genes in G. hirsutum and G. barbadense could be classified into five groups based on the evolutionary tree (Figure 1), similar results have also been found in Arabidopsis and Populus [12]. As shown in Figure 1, cotton CIPK genes were more closely related with AtCIPKs than OsCIPKs, which was consistent with the evolutionary relationships among cotton, Arabidopsis, and rice. Gene structure can provide important information on gene family evolution [54,55]. Cotton CIPKs were clearly divided into exon-rich (Group I) and exon-poor (Group II, III, IV, and V) clades based on their gene structures (Figure 2); some similar results for CIPKs were also observed in Arabidopsis, poplar, and soybean $[12,14]$. Combined with the evolution analysis of CIPK in plants [14], suggests that intron 
gain or loss events were the major driving factors for the gene structural evolution of the CIPK gene family before eudicot-monocot divergence. CIPK genes were unevenly distributed on chromosomes of the two allotetraploid cotton species (Figure 3); it indicates that this might be caused by differential rates of genomic evolution and intergenomic hereditary information transfer [56,57].

Allotetraploid cotton species (G. hirsutum and G. barbadense) resulted from the hybridization between two putative diploid cotton species (A-genome-like African diploid and D-genome-like American diploid) [58]. A total of 80 and 78 CIPK genes were identified in G. hirsutum and G. barbadense, respectively (Tables S2 and S3). The number of CIPK genes in each of the G. hirsutum and G. barbadense genome was greater than that in Arabidopsis (26) and rice (34), and was basically equal to the total sum of G. raimondii (41) and G. arboretum (39), suggesting that the CIPK gene family expands during evolution. G. raimondii and G. arboretum underwent a Gossypium-specific whole-genome duplication (WGD) $[27,30,31]$, and the CIPK gene family of G. raimondii and G. arboretum may expand by an ancient WGD event. Therefore, the expansion of the CIPK gene family in G. hirsutum and G. barbadense may be due to the hybridization and subsequent polyploidization event. Meanwhile, duplicated CIPKS in G. hirsutum and G. barbadense were located on different chromosomes and segmental duplication events were important for the expansion of the CIPK gene family in G. hirsutum and G. barbadense (Figure 3 and Table S7). Therefore, we speculated that the expansion of the CIPK gene family in the two cotton species was mainly due to WGD/segmental duplications. Gene duplication plays a key role in the process of gene family expansion, and plants rapidly adapt to new environments after segmental duplication and translocation [59]. Most of the duplicated CIPK genes in G. hirsutum and G. barbadense were driven by purifying selection as indicated by the Ka/Ks ratio $<1$ (Table 1 ), suggesting that the functions of the duplicated cotton CIPK genes were highly conserved during subsequent evolutionary events, which could eliminate deleterious loss-of-function mutations, and a new duplicated gene at both duplicate loci could be fixed and enhanced after purifying selection [60].

\subsection{Expression Patterns of GhCIPK Gene Family and the Role of GhCIPK6}

CIPK genes are involved in plant growth and development, oil synthesis, and response to various stresses $[16,17,29]$. In many plants, the spatiotemporal expression profiles of CIPK genes have already been reported, including apple, cassava, Arabidopsis, B. napus, and wheat. The MdCIPK genes were highly expressed in root, flower, and fruit [16]. Additionally, some CIPK genes were expressed abundantly in certain tissues (root, flag leaf) in wheat [18]. In the present study, some GhCIPK genes were constitutively expressed in our tested tissues, and some GhCIPK genes were expressed abundantly in certain tissues (Figure 4), indicating that these genes may play important roles in the growth and development of cotton plants. It is worth noting that 39 pairs of homoelogs for GhCIPK genes showed very similar gene structure in terms of exon number and intron length (Figure 2). Among the 109 duplicated gene pairs in G. hirsutum, 69 pairs of duplicated GhCIPKs showed similar expression patterns (Figure 4), while the expression patterns of others were divergent. The differential expression patterns of duplicated CIPK genes suggests that they may have experienced functional divergence. After gene duplication, the new gene could be considered a redundant gene compared with the existing gene, and it can be considered a driving force for evolutionary innovation [61]. Inserting or deleting tissue-specific enhancers or repressors in the coding regions of duplicated genes could obtain a new regulatory context, which might be the cause of functional divergence [62]. Overexpression of GhCIPK6 in cotton could reduce oil content, and increased C18:1 and C18:1+C18:1d6 in transgenic cotton lines, as compared to wild-type plants (Figure 7), indicated that GhCIPK6 plays a negative role in oil synthesis. Similar results could also be presented in the function of SNF1, BnCIPK9, and AtCIPK9 $[5,29]$.

\subsection{Putative Molecular Regulatory Mechanisms of GhCIPKs in Cotton}

In the present study, we predicted the TFBSs in the $1.5 \mathrm{~kb}$ promoter sequence regions of 80 GhCIPKs and found that the 28 different TFs families (Table S9) might bind to GhCIPKs and potentially regulate CIPK genes' expression. TFs regulate transcriptional initiation and control multiple cellular processes 
and are fundamental in plant development and environmental response [63]. In wheat, Luang et al. found that a SnRK3/CIPK family member could interact with TabZIP2, a $b Z I P$ TF, and promote its activity [64]. Recently, an integrative picture was drawn, which showed that different SnRKs and TOR kinase were highly interconnected to control nutrient and stress responses of plants [65]. In apple, an apple CIPK protein kinase, MdCIPK22, targeted a novel residue of AREB transcription factor, Thr411, for ABA-induced phosphorylation, and in the ABA signaling pathway, this was a novel phosphorylation site in the CIPK-AREB regulatory module [66]. In Arabidopsis, abscisic acid repressor 1 (ABR1) was identified as the downstream target of CIPK3, and CIPK3 interacted with ABR1 to regulate ABA response during seed germination [67]. However, how cotton CIPK genes are regulated by these TFs involved in various stresses or plant growth and development remains unknown; this needs to be investigated through the following research using newly developed technologies.

Protein-coding genes may be negatively regulated by a type of miRNA, which plays an important role in a variety of processes, such as response to environmental stress and plant growth and development $[53,68,69]$. Many miRNAs were identified in cotton, and some of them were differentially expressed in different tissues or under various abiotic stresses [68,70,71]. For example, 65 conserved miRNA families were identified from small RNA-seq of cotton leaf and ovule, and 32 families were expressed differentially between cotton leaf and ovule [68]. A total of 113 miRNAs, containing 111 miRNAs and two novel miRNAs, were identified in Xuzhou 142 and its fuzzless/lintless mutant in 0-10 DNA ovules [72]. Overexpression of miR157 could suppress the expression of SQUAMOSA promoter-binding protein-like (SPL) genes and lead to smaller floral organs, fewer ovules, and decreased seed production [73]. In cotton, 319 known miRNAs and 800 novel miRNAs were identified under lowand high-temperature stresses, and almost one-third of the temperature-responsive miRNA-targeted TFs were shown to be related to the regulation of plant growth and development, including the CIPK family [74]. However, there remains a lack of information about miRNA-guided regulation of cotton CIPK genes at the post-transcriptional level in cotton growth and development. In our study, we found three ghr-miRNAs (novel_mir_54, ghr-miR7498, and novel_mir_27) and their target GhCIPK mRNAs (GhCIPK5, GhCIPK6, and GhCIPK58) showed negative regulatory relationships in cotton ovules by qRT-PCR (Figure S10). This implies that the three cotton miRNAs may play important roles in cotton ovules by regulating GhCIPK5, GhCIPK6, and GhCIPK58, respectively. Our findings suggest that some GhCIPKs could be regulated by upland cotton miRNAs, but the true regulatory relationships between miRNAs and GhCIPKs need further study.

\section{Materials and Methods}

\subsection{Identification of CIPK Genes in G. hirsutum and G. barbadense}

Genome assemblies of G. hirsutum acc. TM-1 from Nanjing Agricultural University (NAU version), Zhejiang University (ZJU version,) and Huazhong Agricultural University (HAU version) were downloaded from the Cotton Functional Genomics Database (CottonFGD) (https://cottonfgd.org/) [75], and three genome files of G. barbadense, acc. 3-79 (HAU version), cv. Xinhai 21 (NAU version), and cv. Hai7124 (ZJU version) were also downloaded from CottonFGD (https://cottonfgd.org/). The published CIPK proteins of Arabidopsis [9] and rice [10] were downloaded from the TAIR (https://www. arabidopsis.org/index.jsp) database and the rice genome database (http://rice.plantbiology.msu.edu//), respectively (Table S1). Afterwards, they were used as queries to search against G. hirsutum and G. barbadense genome databases with BLASTP program with an $E$-value $<1 \times 10^{-5}$. Then, each cotton CIPK protein was subjected to the InterProscan (http://www.ebi.ac.uk/Tools/pfa/iprscan/) [76] and SMART (http://smart.embl-heidelberg.de/) [77] databases to confirm the presence of the protein kinase domain (PF00069) and the NAF domain (PF03822). The details on GhCIPK and GbCIPK genes were obtained from CottonFGD (https://cottonfgd.org/). 


\subsection{Phylogenetic Analysis and Synteny Analysis}

The protein sequences of all the identified CIPKs from G. hirsutum, G. barbadense, Arabidopsis, and rice were aligned using the Clustal $\mathrm{X}$ v2.0 program [78]. A phylogenetic tree was constructed using the neighbor-joining (NJ) method in MEGA 5.0 software [79] with poisson correction model. The classification of GhCIPKs and GbCIPKs was consistent with the previous classification reported in Arabidopsis and poplar [12]. The gene structures of cotton CIPKs were graphically visualized using the GSDS (Gene Structure Display Server) tool [80]. The MEME program (http://meme-suite.org/tools/ meme) was used to analyze the motifs of cotton CIPK proteins. The number of repetitions was set as any, the optimum width of motifs ranged from 6 to 200 residues, the maximum number of motifs was 18 , and the other default parameter settings were used.

The chromosomal location images of cotton CIPKs were visualized with Mapchart v2.2 [81] and Circos-0.67 (http://circos.ca/). Orthologous and homoeologs for CIPK genes were identified among the genomes of the diploid and allotetraploid cotton species based on phylogenetic trees and sequence alignments [48,82]. The gene duplication events of two allotetraploid cotton species were analyzed by using MCScanX software, and according to the length of aligned sequence, covered $>80 \%$ between aligned gene sequences, similarly to the aligned regions (>80\%) [48]. DnaSP v5.0 software [83] was used to calculate the synonymous substitution (Ks) and nonsynonymous substitution (Ka).

\subsection{Prediction of Cis-Elements and Transcription Factor Binding Sites in the Promoter Region}

The genomic sequences $1.5 \mathrm{~kb}$ upstream of the start codons of 80 GhCIPKs were extracted, and their regulation elements were predicted using the PlantCARE database (http://bioinformatics.psb.ugent.be/ webtools/plantcare/html/). The putative transcription factor binding sites (TFBSs) of the GhCIPK gene promoter regions were predicted using the PlantTFDB 4.0 server (http://planttfdb.cbi.pku.edu.cn/) with a stricter parameter: threshold $p$-value $\leq 1 \times 10^{-7}$.

\subsection{Alternative Splicing Events Analysis and Potential microRNA Target Analysis}

Alternative splicing (AS) events of GhCIPKs were identified from our mRNA-seq data of cotton floral buds, and AS events were classified into alternative $3^{\prime}$ acceptor sites (AA), alternative $5^{\prime}$ donor sites (AD), intron retention (IR), and exon skipping (ES).

Upland cotton microRNA (miRNA) sequences were obtained from the plant MicroRNA database (http://bioinformatics.cau.edu.cn/PMRD/), miRBase (http://www.mirbase.org/) and published articles $[53,70]$. GhCIPK genes targeted by miRNAs were predicted by searching the coding sequence regions of 80 GhCIPKs for complementary sequences using the online psRNATarget server (http://plantgrn.noble.org/psRNATarget/home) with default parameters as described in a reference [78].

\subsection{Plant Growth Conditions and Treatments}

Cotton seedlings of TM- 1 were grown in a plant growth chamber at $28 \pm 2{ }^{\circ} \mathrm{C}$ under a $16 \mathrm{~h} \mathrm{light} / 8$ $\mathrm{h}$ dark cycle. The third true leaf stage seedlings were treated with salt stress or cold stress. Seedlings were grown in Hoagland nutrient solution and treated with 150, 200, and $300 \mathrm{mM} \mathrm{NaCl}$ for $24 \mathrm{~h}$ and treated with water for $24 \mathrm{~h}$ as a control condition. Seedlings were incubated at $4{ }^{\circ} \mathrm{C}$ for 0,12 , and $24 \mathrm{~h}$ in a temperature-controlled chamber. The leaves at each time point of each treatment were harvested and immediately frozen with liquid nitrogen and stored at $-80^{\circ} \mathrm{C}$ for subsequent RNA isolation.

\subsection{RNA Isolation and Expression Profiling Analysis}

The RNAprep Pure Plant Kit (Tiangen, Beijing, China) was used to extract total RNA from all samples. Approximately $500 \mathrm{ng}$ of RNA was reverse transcribed to cDNA using the PrimerScript 1st Strand cDNA Synthesis Kit (TaKaRa, Dalian, China). Reverse transcription polymerase chain reaction (RT-PCR) was used to validate the accuracy of AS events, and the reaction was performed at $50{ }^{\circ} \mathrm{C}$ for $30 \mathrm{~min}, 95^{\circ} \mathrm{C}$ for $2 \mathrm{~min}$, followed by 29 cycles at $94^{\circ} \mathrm{C}$ for $30 \mathrm{~s}, 60^{\circ} \mathrm{C}$ for $30 \mathrm{~s}$, and $72{ }^{\circ} \mathrm{C}$ for $35 \mathrm{~s}$, 
followed by $72{ }^{\circ} \mathrm{C}$ for a 2 min extension step. The gene-specific primers for qRT-PCR and RT-PCR were designed using Primer v5.0 software, and primers for RT-PCR were designed to span the splicing events. All primers are listed in Table S11. GhUBQ7 was used as an internal control. The qRT-PCR was strictly performed with SYBR Premix Ex Taq Kit (TaKaRa) according to the manufacturer's instructions, and qRT-PCR was performed at $95^{\circ} \mathrm{C}$ for $2 \mathrm{~min}$, followed by 40 cycles of $95^{\circ} \mathrm{C}$ for $5 \mathrm{~s}, 58^{\circ} \mathrm{C}$ for $20 \mathrm{~s}$, and $72{ }^{\circ} \mathrm{C}$ for $30 \mathrm{~s}$ in a 96-well plate. An ABI 7500 real-time PCR system (Applied Biosystems, Foster City, CA, USA) was used to run all qRT-PCRs. Three biological replicates and three technical replicates were conducted for each sample. The relative expression levels of GhCIPK genes were calculated by the $2^{-\Delta \Delta C t}$ method [52].

The expression value (FPKMs, fragments per kilobase per million reads) of the GhCIPK gene family was extracted from the RNA-seq data in various G. hirsutum acc. TM-1 tissues (PRJNA248163) [34]. Gene expression levels were calculated as FPKM. MeV 4.0 software was used to generate heat maps of the GhCIPK genes.

\subsection{Identification of Single Nucleotide Polymorphisms (SNPs) for CIPK Genes and Correlation Analysis with Cottonseed Oil and Protein Content Traits}

To identify the single nucleotide polymorphism (SNPs) for CIPK genes, the RNA-seq data from CRI 36 and Hai 7124 was analyzed, and SNPs were scanned using SOAPsnp software [84]. CRI 36 is currently a commercial cultivar of G. hirsutum and Hai 7124 is a non-commercial G. barbadense. We used an interspecific backcross inbred line (BIL) population of 180 lines derived from CRI 36 and Hai 7124 to validate SNP markers of cotton CIPKs. SNP primers for CIPK genes were designed using Primer v5.0 software (Table S11), and high-resolution melting (HRM) was used to analyze the relationship of SNPs and cottonseed oil content as described in previous articles [48]. The simple correlation analysis was performed using SPPS software (IBM, New York, NY, USA).

\subsection{Genetic Transformation, Oil Content Detection, and Fatty Acid Composition Analysis}

Combined with RT-PCR and rapid amplification of cDNA ends technique (RACE), the full length of GhCIPK6 was obtained from 'ZG5' cDNA. The plant binary vector pBI121, which carried the NPT II (neomycin phosphotransferase II) gene, was used for expression of the GhCIPK6 gene under the control of the CaMV 35S promoter. The '11-0516' upland cotton cultivar was grown in the field until flowering, and after self-pollination for $24 \mathrm{~h}$, the plasmid of recombinant vector (pBI121-GhCIPK6) was injected into the ovary of ' $11-0516$ ' by syringe. These injected mature seeds were grown again, and positive transgenic plants were detected using the method described in previous reports $[85,86]$. According to the manufacturer's instructions of DIG High Prime DNA Labeling and Detection Starter kit I (Roche, Basel, Switzerland), Southern blotting was conducted using the NPT II gene as the DNA probe to detect GhCIPK6 copies.

The seed oil contents of transgenic cotton plants and wild-type plants were detected in approximately $1 \mathrm{~g}$ of seeds of each sample by an NMI20-Analyst nuclear magnetic resonance spectrometer (Niumag, Shanghai, China). The fatty acid composition of each cotton sample was analyzed using a gas chromatograph (GC-2030, Shimadzu, Kyoto, Japan) equipped with a flame ionization detector (FID) and an SH-Rtx-65 capillary column $(30 \mathrm{~m} \times 0.25 \mathrm{~mm} \times 0.50 \mu \mathrm{m})$. Samples $(1 \mu \mathrm{L})$, including fatty acids and the n-hexane solution, were injected at $280{ }^{\circ} \mathrm{C}$ in split mode $(30: 1)$. All oil content and fatty acid composition data were analyzed by Student's $t$-test. Differences were considered statistically significant when $p<0.05\left(^{*}\right)$ compared with the control.

\section{Conclusions}

In the present study, a total of 158 CIPK genes from G. hirsutum and G. barbadense were identified. Among them, phylogenetic classifications, gene structures, motifs, chromosomal localizations, and duplication genes were analyzed. Segmental duplication was a major impetus for the expansion of cotton CIPK gene family, and purifying selection played a major role in the evolution of the gene 
family. Additionally, cis-elements, expression patterns, and co-localization relationship of GhCIPK genes with oil or protein QTLs were also analyzed. Most of GhCIPK genes were not associated with natural variations in cotton oil content. Overexpression of GhCIPK6 gene could reduce oil content and change fatty acid composition. Additionally, the relationships of miRNAs and their target GhCIPK genes showed that three genes were regulated by miRNAs. Our results provide a solid foundation for further studies of the roles of CIPK genes in stress responses and oil synthesis.

Supplementary Materials: Supplementary materials can be found at http://www.mdpi.com/1422-0067/21/3/863/s1. Table S1. The CIPK genes used to construct phylogenetic tree in other species. Table S2. The list of the CIPK genes identified in G. hirsutum. Table S3. The list of the CIPK genes identified in G. barbadense. Table S4. Detailed information about the protein kinase domain and the NAF domain in each cotton CIPK protein. Table S5. Orthologous CIPK genes in and between the four cotton species. Table S6. Homoeologs for CIPK genes in the two allotetraploid cotton species. Table S7. Duplication of CIPK genes in G. hirsutum and G. barbadense. Table S8. The list of cis-elements in the $1.5 \mathrm{~kb}$ promoter regions of GhCIPKs. Table S9. The list of TFs in the $1.5 \mathrm{~kb}$ promoter regions of GhCIPKs. Table S10. The information of 17 non-synonymous SNPs in G. hirsutum and G. barbadense. Table S11. The list of primers in our study. Table S12. Association analysis between SNP markers and cottonseed oil content in a BIL population. Table S13. The predicted details of miRNA-mediated targeting regulatory relations with GhCIPK gene family in cotton. Figure S1. The distribution of orthologous CIPK gene pairs in and between G. raimondii, G. arboreum, G. hirsutum, and G. barbadense. Figure S2. The distribution of homoeologs for CIPK gene pairs between G. hirsutum and G. barbadense. Figure S3. Phylogenetic relationship of CIPK genes from G. raimondii, G. arboretum, and G. hirsutum. Figure S4. Phylogenetic relationship of CIPK genes from G. raimondii, G. arboretum, and G. barbadense. Figure S5. Conserved motifs in the CIPK proteins in G. hirsutum and G. barbadense. The MEME program was used on all motifs of CIPKs from G. hirsutum and G. barbadense. Each colored box represents a motif in cotton CIPK proteins. Figure S6. Sequence logos of cotton CIPK proteins. Figure S7. The NAF domain in the CIPK proteins. The four cotton CIPK proteins were compared using the DNAMAN 7.0 software and conserved amino acid residues are shown in dark blue. Figure S8. A co-localization analysis of GhCIPK genes with cotton oil and protein content QTLs. GhCIPK genes are shown in red and underlining indicates the GhCIPK genes colocalized with oil or protein content QTLs. QTLs from the CottonQTL database $[45,46]$ are indicated by black color, and QTLs from the recently published article [47] are indicated by red color. Figure S9. Characterization of alternative splicing (AS) events and validation of full-length isoforms using RT-PCR. (A) Classification of AS events; (B) RT-PCR validation of AS events for GhCIPK17. Figure S10. The relative expression levels of miRNAs and their putative targeting GhCIPKs in cotton ovules. Figure S11. Potential alternative spliced isoform structure of GhCIPK48 and GhCIPK51 targeted by cotton miRNAs. The dark red and red dotted rectangles were the predicted miRNA targeting sites. Exons are represented by green boxes and introns by dark lines.

Author Contributions: Y.C. and J.Y. conceived and designed the experiments. J.W. and B.J. prepared the figures and tables. Y.S., M.W. and W.P. analyzed and interpreted the data. Y.C. prepared the manuscript. J.Z. revised the manuscript. All authors have read and agreed to the published version of the manuscript.

Funding: This research was mainly supported by National Key Research and Development Program of China (2018YFD0100301) and China Postdoctoral Science Foundation (Grant 2019M650916).

Acknowledgments: We are grateful to Guoyuan Liu and Bingbing Zhang (Cotton Institute of the Chinese Academy of Agricultural Sciences) for their helpful suggestions on the miRNA analysis of our manuscript.

Conflicts of Interest: The authors declare no conflict of interest.

\section{References}

1. Luan, S. The CBL-CIPK network in plant calcium signaling. Trends Plant Sci. 2009, 14, 37-42. [CrossRef] [PubMed]

2. Kudla, J.; Batistič, O.; Hashimoto, K. Calcium signals: The lead currency of plant information processing. Plant Cell 2010, 22, 541-563. [CrossRef] [PubMed]

3. Huang, C.; Ding, S.; Zhang, H.; Du, H.; An, L. CIPK7 is involved in cold response by interacting with CBL1 in Arabidopsis thaliana. Plant Sci. 2011, 181, 57-64. [CrossRef] [PubMed]

4. Halford, N.G.; Hey, S.; Jhurreea, D.; Laurie, S.; Mckibbin, R.S.; Paul, M.; Zhang, Y. Metabolic signalling and carbon partitioning: Role of Snf1-related (SnRK1) protein kinase. J. Exp. Bot. 2003, 54, 467-475. [CrossRef]

5. John, S.; Raymond, J.; Hongxian, H.; Quinn, Z.; Seung-Pyo, H. Snf1 is a regulator of lipid accumulation in Yarrowia lipolytica. Appl. Environ. Microbiol. 2013, 79, 7360-7370.

6. Albrecht, V.; Ritz, O.; Linder, S.; Harter, K.; Kudla, J. The NAF domain defines a novel protein-protein interaction module conserved in $\mathrm{Ca}^{2+}$-regulated kinases. EMBO J. 2014, 20, 1051-1063. [CrossRef]

7. Guo, Y.; Halfter, U.; Ishitani, M.; Zhu, J.K. Molecular characterization of functional domains in the protein kinase SOS2 that is required for plant salt tolerance. Plant Cell 2001, 13, 1383-1399. [CrossRef] 
8. Masaru, O.; Yan, G.; Ursula, H.; Jian-Kang, Z. A novel domain in the protein kinase SOS2 mediates interaction with the protein phosphatase 2C ABI2. Proc. Natl. Acad. Sci. USA 2003, 100, 11771-11776.

9. Kolukisaoglu, Ü.; Weinl, S.; Blazevic, D.; Batistic, O.; Kudla, J. Calcium sensors and their interacting protein kinases: Genomics of the Arabidopsis and rice CBL-CIPK signaling networks. Plant Physiol. 2004, 134, 43-58. [CrossRef]

10. Kanwar, P.; Sanyal, S.K.; Tokas, I.; Yadav, A.K.; Pandey, A.; Kapoor, S.; Pandey, G.K. Comprehensive structural, interaction and expression analysis of CBL and CIPK complement during abiotic stresses and development in rice. Cell Calcium. 2014, 56, 81-95. [CrossRef]

11. Chen, X.; Gu, Z.; Xin, D.; Hao, L.; Liu, C.; Huang, J.; Ma, B.; Zhang, H. Identification and characterization of putative CIPK genes in maize. J. Genet. Genom. 2011, 38, 77-87. [CrossRef] [PubMed]

12. Yu, Y.; Xia, X.; Yin, W.; Zhang, H. Comparative genomic analysis of CIPK gene family in Arabidopsis and Populus. Plant Growth Regul. 2007, 52, 101-110. [CrossRef]

13. Zhang, H.; Yang, B.; Liu, W.Z.; Li, H.; Wang, L.; Wang, B.; Deng, M.; Liang, W.; Deyholos, M.K.; Jiang, Y.Q. Identification and characterization of CBL and CIPK gene families in canola (Brassica napus L.). BMC Plant Biol. 2014, 14, 8. [CrossRef] [PubMed]

14. Zhu, K.; Chen, F.; Liu, J.; Chen, X.; Hewezi, T.; Cheng, Z.M. Evolution of an intron-poor cluster of the CIPK gene family and expression in response to drought stress in soybean. Sci. Rep. 2016, 6, 28225. [CrossRef] [PubMed]

15. Xi, Y.; Liu, J.; Dong, C.; Cheng, Z.M. The CBL and CIPK gene family in grapevine (Vitis vinifera): Genome-wide analysis and expression profiles in response to various abiotic stresses. Front. Plant Sci. 2017, 8, 978. [CrossRef] [PubMed]

16. Niu, L.; Dong, B.; Song, Z.; Dong, M.; Fu, Y. Genome-wide identification and characterization of CIPK family and analysis responses to various stresses in apple (Malus domestica). Int. J. Mol. Sci. 2018, 19, 2131. [CrossRef] [PubMed]

17. Li, P.; Zheng, T.; Li, L.; Zhuo, X.; Jiang, L.; Wang, J.; Cheng, T.; Zhang, Q. Identification and comparative analysis of the CIPK gene family and characterization of the cold stress response in the woody plant Prunus mume. Peer J. 2019, 7, e6847. [CrossRef] [PubMed]

18. Sun, T.; Wang, Y.; Wang, M.; Li, T.; Zhou, Y.; Wang, X.; Wei, S.; He, G.; Yang, G. Identification and comprehensive analyses of the CBL and CIPK gene families in wheat (Triticum aestivum L.). BMC Plant Biol. 2015, 15, 269. [CrossRef]

19. Wang, J.; Lu, X.; Yin, Z.; Mu, M.; Zhao, X.; Wang, D.; Wang, S.; Fan, W.; Guo, L.; Ye, W. Genome-wide identification and expression analysis of CIPK genes in diploid cottons. Genet. Mol. Res. 2016, 15, 10.4238. [CrossRef]

20. Kim, K.N.; Cheong, Y.H.; Grant, J.J.; Pandey, G.K.; Luan, S. CIPK3, a calcium sensor-associated protein kinase that regulates abscisic acid and cold signal transduction in Arabidopsis. Plant Cell 2003, 15, 411-423. [CrossRef]

21. He, L.; Yang, X.; Wang, L.; Zhu, L.; Zhou, T.; Deng, J.; Zhang, X. Molecular cloning and functional characterization of a novel cotton CBL-interacting protein kinase gene (GhCIPK6) reveals its involvement in multiple abiotic stress tolerance in transgenic plants. Biochem. Biophys. Res. Commun. 2013, 435, $209-215$. [PubMed]

22. Tai, F.; Yuan, Z.; Li, S.; Qi, W.; Liu, F.; Wei, W. ZmCIPK8, a CBL-interacting protein kinase, regulates maize response to drought stress. Plant Cell Tiss. Org. Cul. 2016, 124, 459-469. [CrossRef]

23. Wang, Y.; Li, T.; John, S.J.; Chen, M.; Chang, J.; Yang, G.; He, G. A CBL-interacting protein kinase TaCIPK27 confers drought tolerance and exogenous ABA sensitivity in transgenic Arabidopsis. Plant Physiol. Biochem. 2017, 123, 103-113. [CrossRef] [PubMed]

24. Pan, W.; Shen, J.; Zheng, Z.; Yan, X.; Shou, J.; Wang, W.; Jiang, L.; Pan, J. Overexpression of the Tibetan Plateau annual wild barley (Hordeum spontaneum) HsCIPKs enhances rice tolerance to heavy metal toxicities and other abiotic stresses. Rice 2018, 11, 51. [CrossRef] [PubMed]

25. Aslam, M.; Fakher, B.; Anandhan, S.; Pande, V.; Ahmed, Z.; Qin, Y. Ectopic expression of cold responsive LlaCIPK gene enhances cold stress tolerance in Nicotiana tabacum. Genes 2019, 10, 446. [CrossRef] [PubMed]

26. Shang, L.; Abduweli, A.; Wang, Y.; Hua, J. Genetic analysis and QTL mapping of oil content and seed index using two recombinant inbred lines and two backcross populations in Upland cotton. Plant Breed. 2016, 135, 224-231. [CrossRef] 
27. Paterson, A.H.; Wendel, J.F.; Heidrun, G.; Hui, G.; Jerry, J.; Dianchuan, J.; Danny, L.; Showmaker, K.C.; Shengqiang, S.; Joshua, U.; et al. Repeated polyploidization of Gossypium genomes and the evolution of spinnable cotton fibres. Nature 2012, 492, 423-427. [CrossRef]

28. Ma, J.; Liu, J.; Pei, W.; Ma, Q.; Wang, N.; Zhang, X.; Cui, Y.; Li, D.; Liu, G.; Wu, M.; et al. Genome-wide association study of the oil content in upland cotton (Gossypium hirsutum L.) and identification of GhPRXR1, a candidate gene for a stable QTLqOC-Dt5-1. Plant Sci. 2019, 286, 89-97. [CrossRef]

29. Guo, Y.; Huang, Y.; Gao, J.; Pu, Y.; Wang, N.; Shen, W.; Wen, J.; Yi, B.; Ma, C.; Tu, J. CIPK9 is involved in seed oil regulation in Brassica napus L. and Arabidopsis thaliana (L.) Heynh. Biotechnol. Biofuels 2018, 11, 124. [CrossRef]

30. Wang, K.; Wang, Z.; Li, F.; Ye, W.; Wang, J.; Song, G.; Yue, Z.; Cong, L.; Shang, H.; Zhu, S.; et al. The draft genome of a diploid cotton Gossypium raimondii. Nat. Genet. 2012, 44, 1098-1103.

31. Li, F.; Fan, G.; Wang, K.; Sun, F.; Yuan, Y.; Song, G.; Li, Q.; Ma, Z.; Lu, C.; Zou, C.; et al. Genome sequence of the cultivated cotton Gossypium arboreum. Nat. Genet. 2014, 46, 567-572. [CrossRef] [PubMed]

32. Du, X.; Huang, G.; He, S.; Yang, Z.; Sun, G.; Ma, X.; Li, N.; Zhang, X.; Sun, J.; Liu, M.; et al. Resequencing of 243 diploid cotton accessions based on an updated A genome identifies the genetic basis of key agronomic traits. Nat. Genet. 2018, 50, 796-802. [CrossRef] [PubMed]

33. Li, F.; Fan, G.; Lu, C.; Xiao, G.; Zou, C.; Kohel, R.J.; Ma, Z.; Shang, H.; Ma, X.; Wu, J.Y.; et al. Genome sequence of cultivated Upland cotton (Gossypium hirsutum TM-1) provides insights into genome evolution. Nat. Biotechnol. 2015, 33, 524-530. [CrossRef] [PubMed]

34. Zhang, T.; Hu, Y.; Jiang, W.; Fang, L.; Guan, X.; Chen, J.; Zhang, J.; Saski, C.A.; Scheffler, B.E.; Stelly, D.M.; et al. Sequencing of allotetraploid cotton (Gossypium hirsutum L. acc. TM-1) provides a resource for fiber improvement. Nat. Biotechnol. 2015, 33, 531-537. [CrossRef]

35. Hu, Y.; Chen, J.; Fang, L.; Zhang, Z.; Ma, W.; Niu, Y.; Ju, L.; Deng, J.; Zhao, T.; Lian, J.; et al. Gossypium barbadense and Gossypium hirsutum genomes provide insights into the origin and evolution of allotetraploid cotton. Nat. Genet. 2019, 51, 739-748. [CrossRef]

36. Wang, M.; Tu, L.; Yuan, D.; Zhu, D.; Shen, C.; Li, J.; Liu, F.; Pei, L.; Wang, P.; Zhao, G.J.; et al. Reference genome sequences of two cultivated allotetraploid cottons, Gossypium hirsutum and Gossypium barbadense. Nat. Genet. 2019, 51, 224-229. [CrossRef]

37. Liu, X.; Zhao, B.; Zheng, H.-J.; Hu, Y.; Lu, G.; Yang, C.-Q.; Chen, J.-D.; Chen, J.-J.; Chen, D.-Y.; Zhang, L.; et al. Gossypium barbadense genome sequence provides insight into the evolution of extra-long staple fiber and specialized metabolites. Sci. Rep. 2015, 5, 14139. [CrossRef]

38. Yuan, D.; Tang, Z.; Wang, M.; Gao, W.; Tu, L.; Jin, X.; Chen, L.; He, Y.; Zhang, L.; Zhu, L.; et al. The genome sequence of Sea-Island cotton (Gossypium barbadense) provides insights into the allopolyploidization and development of superior spinnable fibres. Sci. Rep. 2015, 5, 17662. [CrossRef]

39. Wang, W.; Cheng, Y.; Chen, D.; Liu, D.; Hu, M.; Dong, J.; Zhang, X.; Song, L.; Shen, F. The catalase gene family in cotton: Genome-wide characterization and bioinformatics analysis. Cells 2019, 8, 86. [CrossRef]

40. Ma, J.; Liu, F.; Wang, Q.; Wang, K.; Jones, D.C.; Zhang, B. Comprehensive analysis of TCP transcription factors and their expression during cotton (Gossypium arboreum) fiber early development. Sci. Rep. 2016, 6, 21535. [CrossRef]

41. Liu, J.; Osbourn, A.; Ma, P. MYB transcription factors as regulators of phenylpropanoid metabolism in plants. Mol. Plant 2015, 8, 689-708. [CrossRef] [PubMed]

42. Noman, A.; Aqeel, M.; Khalid, N.; Islam, W.; Sanaullah, T.; Anwar, M.; Khan, S.; Ye, W.; Lou, Y. Zinc finger protein transcription factors: Integrated line of action for plant antimicrobial activity. Microb. Pathog. 2019, 132, 141-149. [CrossRef]

43. Mahapatra, M.; Mahanty, B.; Joshi, R.K. Genome wide identification and functional assignments of C2H2 Zinc-finger family transcription factors in Dichanthelium oligosanthes. Bioinformation 2019, 15, 689-696. [PubMed]

44. Lyzenga, W.J.; Liu, H.; Schofield, A.; Muise-Hennessey, A.; Stone, S.L. Arabidopsis CIPK26 interacts with KEG, components of the ABA signalling network and is degraded by the ubiquitin-proteasome system. J. Exp. Bot. 2013, 64, 2779-2791. [CrossRef]

45. Said, J.I.; Lin, Z.; Zhang, X.; Song, M.; Zhang, J. A comprehensive meta QTL analysis for fiber quality, yield, yield related and morphological traits, drought tolerance, and disease resistance in tetraploid cotton. BMC Genom. 2013, 14, 776. [CrossRef] 
46. Said, J.I.; Song, M.; Wang, H.; Lin, Z.; Zhang, X.; Fang, D.D.; Zhang, J. A comparative meta-analysis of QTL between intraspecific Gossypium hirsutum and interspecific G. hirsutum $\times$ G. barbadense populations. Mol. Genet. Genom. 2015, 290, 1003-1025. [CrossRef]

47. Ma, J.; Pei, W.; Ma, Q.; Geng, Y.; Liu, G.; Liu, J.; Cui, Y.; Zhang, X.; Wu, M.; Li, X.; et al. QTL analysis and candidate gene identification for plant height in cotton based on an interspecific backcross inbred line population of Gossypium hirsutum $\times$ Gossypium barbadense. Theor. Appl. Genet. 2019, 132, 2663-2676. [CrossRef]

48. Cui, Y.; Ma, J.; Liu, G.; Wang, N.; Pei, W.; Wu, M.; Li, X.; Zhang, J.; Yu, J. Genome-wide identification, sequence variation and expression of the glycerol-3-phosphate acyltransferase (GPAT) gene family in Gossypium. Front. Genet. 2019, 10, 116. [CrossRef]

49. Laloum, T.; Martín, G.; Duque, P. Alternative splicing control of abiotic stress responses. Trends Plant Sci. 2018, 23, 140-150. [CrossRef]

50. Marquez, Y.; Brown, J.W.S.; Simpson, C.; Barta, A.; Kalyna, M. Transcriptome survey reveals increased complexity of the alternative splicing landscape in Arabidopsis. Genome Res. 2012, 22, 1184-1195. [CrossRef]

51. Wang, M.; Wang, P.; Liang, F.; Ye, Z.; Li, J.; Shen, C.; Pei, L.; Wang, F.; Hu, J.; Tu, L.; et al. A global survey of alternative splicing in allopolyploid cotton: Landscape, complexity and regulation. New Phytol. 2018, 217, 163-178. [CrossRef] [PubMed]

52. Yang, X.; Coulombe-Huntington, J.; Kang, S.; Sheynkman, G.M.; Hao, T.; Richardson, A.; Sun, S.; Yang, F.; Shen, Y.A.; Murray, R.R. Widespread expansion of protein interaction capabilities by alternative splicing. Cell 2016, 164, 805-817. [CrossRef] [PubMed]

53. Zhang, B.; Zhang, X.; Liu, G.; Guo, L.; Qi, T.; Zhang, M.; Li, X.; Wang, H.; Tang, H.; Qiao, X.; et al. A combined small RNA and transcriptome sequencing analysis reveal regulatory roles of miRNAs during anther development of Upland cotton carrying cytoplasmic male sterile Gossypium harknessii (D2) cytoplasm. Bmc Plant Biol. 2018, 18, 242. [CrossRef] [PubMed]

54. Lynch, M. Intron evolution as a population-genetic process. Proc. Natl. Acad. Sci. USA 2002, 99, 6118-6123. [CrossRef] [PubMed]

55. Del Campo, E.M.; Casano, L.M.; Eva, B. Evolutionary implications of intron-exon distribution and the properties and sequences of the RPL10A gene in eukaryotes. Mol. Phylogenet. Evol. 2013, 66, 857-867. [CrossRef] [PubMed]

56. Paterson, A.H.; Bowers, J.E.; Burow, M.D.; Draye, X.; Elsik, C.G.; Jiang, C.-X.; Katsar, C.S.; Lan, T.-H.; Lin, Y.-R.; Ming, R.; et al. Comparative genomics of plant chromosomes. Plant Cell 2000, 12, 1523-1539. [CrossRef] [PubMed]

57. Wendel, J.F.; Cronn, R.C. Polyploidy and the evolutionary history of cotton. Adv. Agron. 2003, 78, 139.

58. Senchina, D.S.; Alvarez, I.; Cronn, R.C.; Liu, B.; Rong, J.; Noyes, R.D.; Paterson, A.H.; Wing, R.A.; Wilkins, T.A.; Wendel, J.F. Rate variation among nuclear genes and the age of polyploidy in Gossypium. Mol. Biol. Evol. 2003, 20, 633-643. [CrossRef]

59. Fraser, J.A.; Huang, J.C.; Pukkila-Worley, R.; Alspaugh, J.A.; Mitchell, T.G.; Heitman, J. Chromosomal translocation and segmental duplication in Cryptococcus neoformans. Eukaryot. Cell 2005, 4, 401-406. [CrossRef]

60. Tanaka, K.M.; Takahasi, K.R.; Takano-Shimizu, T. Enhanced fixation and preservation of a newly arisen duplicate gene by masking deleterious loss-of-function mutations. Genet. Res. 2009, 91, 267-280. [CrossRef]

61. Flagel, L.E.; Wendel, J.F. Gene duplication and evolutionary novelty in plants. New Phytol. 2009, 183, 557-564. [CrossRef] [PubMed]

62. Yang, Z.; Qian, G.; Qin, W.; Yang, Z.; Yuan, C.; Lu, L.; Ge, X.; Zhang, C.; Wu, Z.; Li, F. Genome-wide analysis of WOX genes in upland cotton and their expression pattern under different stresses. BMC Plant Biol. 2017, 17, 113. [CrossRef] [PubMed]

63. Lehti-Shiu, M.D.; Panchy, N.; Wang, P.; Uygun, S.; Shiu, S.-H. Diversity, expansion, and evolutionary novelty of plant DNA-binding transcription factor families. Biochim. Biophys. Acta 2017, 1860, 3-20. [CrossRef] [PubMed]

64. Luang, S.; Sornaraj, P.; Bazanova, N.; Jia, W.; Eini, O.; Hussain, S.S.; Kovalchuk, N.; Agarwal, P.K.; Hrmova, M.; Lopato, S. The wheat TabZIP2 transcription factor is activated by the nutrient starvation-responsive SnRK3/CIPK protein kinase. Plant Mol. Biol. 2018, 96, 543-561. [CrossRef] [PubMed] 
65. Jamsheer, K.M.; Jindal, S.; Laxmi, A. Evolution of TOR-SnRK dynamics in green plants and its integration with phytohormone signaling networks. J. Exp. Bot. 2019, 70, 2239-2259. [CrossRef] [PubMed]

66. Ma, Q.J.; Sun, M.H.; Lu, J.; Liu, Y.J.; You, C.X.; Hao, Y.J. An apple CIPK protein kinase targets a novel residue of AREB transcription factor for ABA-dependent phosphorylation. Plant Cell Environ. 2017, 40, 2207-2219. [CrossRef]

67. Sanyal, S.K.; Kanwar, P.; Yadav, A.K.; Sharma, C.; Kumar, A.; Pandey, G.K. Arabidopsis CBL interacting protein kinase 3 interacts with ABR1, an APETALA2 domain transcription factor, to regulate ABA responses. Plant Sci. 2017, 254, 48-59. [CrossRef]

68. Xie, F.; Jones, D.C.; Wang, Q.; Sun, R.; Zhang, B. Small RNA sequencing identifies miRNA roles in ovule and fibre development. Plant Biotechnol. J. 2015, 13, 355-369. [CrossRef]

69. Hou, J.; Lu, D.; Mason, A.S.; Li, B.; Xiao, M.; An, S.; Fu, D. Non-coding RNAs and transposable elements in plant genomes: Emergence, regulatory mechanisms and roles in plant development and stress responses. Planta 2019, 250, 23-40. [CrossRef]

70. Liu, G.; Wu, M.; Pei, W.; Li, X.; Wang, N.; Ma, J.; Zang, X.; Yu, S.; Zhang, J.; Yu, J. A comparative analysis of small RNAs between two Upland cotton backcross inbred lines with different fiber length: Expression and distribution. Crop J. 2019, 7, 198-208. [CrossRef]

71. Yin, Z.; Li, Y.; Yu, J.; Liu, Y.; Li, C.; Han, X.; Shen, F. Difference in miRNA expression profiles between two cotton cultivars with distinct salt sensitivity. Mol. Biol. Rep. 2012, 39, 4961-4970. [CrossRef] [PubMed]

72. Kwak, P.B.; Wang, Q.Q.; Chen, X.S.; Qiu, C.X.; Yang, Z.M. Enrichment of a set of microRNAs during the cotton fiber development. BMC Genom. 2009, 10, 457. [CrossRef] [PubMed]

73. Liu, N.; Tu, L.; Wang, L.; Hu, H.; Xu, J.; Zhang, X. MicroRNA 157-targeted SPL genes regulate floral organ size and ovule production in cotton. BMC Plant Biol. 2017, 17, 7. [CrossRef] [PubMed]

74. Wang, Q.; Liu, N.; Yang, X.; Tu, L.; Zhang, X. Small RNA-mediated responses to low-and high-temperature stresses in cotton. Sci. Rep. 2016, 6, 35558. [CrossRef]

75. Zhu, T.; Liang, C.; Meng, Z.; Sun, G.; Meng, Z.; Guo, S.; Zhang, R. CottonFGD: An integrated functional genomics database for cotton. BMC Plant Biol. 2017, 17, 101. [CrossRef]

76. Quevillon, E.; Silventoinen, V.; Pillai, S.; Harte, N.; Mulder, N.; Apweiler, R.; Lopez, R. InterProScan: Protein domains identifier. Nucleic Acids Res. 2005, 33, W116-W120. [CrossRef]

77. Ivica, L.; Tobias, D.; Peer, B. SMART: Recent updates, new developments and status in 2015. Nucleic Acids Res. 2015, 43, 257-260.

78. Larkin, M.A.; Blackshields, G.; Brown, N.; Chenna, R.; McGettigan, P.A.; McWilliam, H.; Valentin, F.; Wallace, I.M.; Wilm, A.; Lopez, R.; et al. Clustal W and Clustal X version 2.0. Bioinformatics 2007, 23, 2947-2948. [CrossRef]

79. Tamura, K.; Peterson, D.; Peterson, N.; Stecher, G.; Nei, M.; Kumar, S. MEGA5: Molecular evolutionary genetics analysis using maximum likelihood, evolutionary distance, and maximum parsimony methods. Mol. Biol. Evol. 2011, 28, 2731-2739. [CrossRef]

80. Hu, B.; Jin, J.; Guo, A.-Y.; Zhang, H.; Luo, J.; Gao, G. GSDS 2.0: An upgraded gene feature visualization server. Bioinformatics 2014, 31, 1296-1297. [CrossRef]

81. Voorrips, R. MapChart: Software for the graphical presentation of linkage maps and QTLs. J. Hered. 2002, 93, 77-78. [CrossRef]

82. Li, W.; Ren, Z.; Wang, Z.; Sun, K.; Pei, X.; Liu, Y.; He, K.; Zhang, F.; Song, C.; Zhou, X.; et al. Evolution and stress responses of Gossypium hirsutum SWEET genes. Int. J. Mol. Sci. 2018, 19, 769. [CrossRef] [PubMed]

83. Rozas, J.; Sánchez-DelBarrio, J.C.; Messeguer, X.; Rozas, R. DnaSP, DNA polymorphism analyses by the coalescent and other methods. Bioinformatics 2003, 19, 2496-2497. [CrossRef] [PubMed]

84. Li, R.; Li, Y.; Fang, X.; Yang, H.; Wang, J.; Kristiansen, K.; Wang, J. SNP detection for massively parallel whole-genome resequencing. Genome Res. 2009, 19, 1124-1132. [CrossRef] [PubMed] 
85. Wang, M.; Zhang, B.; Wang, Q. Cotton transformation via pollen tube pathway. Methods Mol. Biol. 2013, 958, 71-77. [PubMed]

86. Yue, Z.; Liu, X.; Zhou, Z.; Hou, G.; Hua, J.; Zhao, Z. Development of a novel-type transgenic cotton plant for control of cotton bollworm. Plant Biotechnol. J. 2016, 14, 1747-1755. [CrossRef] [PubMed] 